\title{
Analytical basis for determining slope lines in grid digital elevation models
}

\author{
Stefano Orlandini, ${ }^{1}$ Giovanni Moretti, ${ }^{1}$ and Andrea Gavioli ${ }^{2}$ \\ Received 19 August 2013; revised 2 December 2013; accepted 12 December 2013; published 22 January 2014.
}

[1] An analytical basis for the determination of slope lines in grid digital elevation models is provided by using the D8-LTD method (eight slope directions, least transverse deviation). The D8-LTD method's capability to predict consistently exact slope lines as the grid cell size goes to zero is shown analytically by applying mathematical analysis methods. The use of cumulative, least transverse deviations is found to be the key factor allowing for globally unbiased approximations of slope lines. The D8-LTD method's properties are also demonstrated numerically by using digital elevation models of a synthetic sloping surface obtained from the Himmelblau function. It is shown that slope lines obtained from the D8LTD method can approximate the exact slope lines as close as desired by selecting a grid cell size that is small enough. In contrast, the standard D8 method is found to produce significantly biased results even when small grid cells are used. The D8-LTD method outperforms the D8 method over a wide range of grid cell sizes (up to $20 \mathrm{~m}$ in this application), beyond which grid cell size becomes too large to validly represent the underlying sloping surface. It is therefore concluded that the D8-LTD method should be used in preference to the standard D8 method in order to obtain slope lines that are only limited in reliability by the detail of topographic data, and not by the accuracy of the slope direction method applied.

Citation: Orlandini, S., G. Moretti, and A. Gavioli (2014), Analytical basis for determining slope lines in grid digital elevation models, Water Resour. Res., 50, 526-539, doi:10.1002/2013WR014606.

\section{Introduction}

[2] Slope lines were defined by Cayley [1859] and Maxwell [1870] as those lines that intersect contour lines at right angles. If overland flows are laminar and purely driven by gravity, then slope lines provide a perfect match to the theoretical flow lines. As also reported by Gallant and Hutchinson [2011], however, a distinction should be made in hydrology and geomorphology between slope lines and flow lines when dispersion is a relevant process as a results of turbulence or diffusional effects. In this case, flow lines and slope lines differ. Multiple flow direction methods were postulated to describe overland flow dispersion [e.g., Freeman, 1991; Quinn et al., 1991; Seibert and McGlynn, 2007]. However, a more cautious modeling philosophy may be adopted by determining slope lines from topographic data with the minimum usage of geomorphologic dispersion and, if necessary, by modeling hydrologic dispersion separately [Tarboton, 1997; Orlandini et al., 2003]. The analysis shown by Orlandini et al. [2012]

\footnotetext{
${ }^{1}$ Dipartimento di Ingegneria "Enzo Ferrari," Università degli Studi di Modena e Reggio Emilia, Modena, Italy.

${ }^{2}$ Dipartimento di Scienze Fisiche, Informatiche e Matematiche, Università degli Studi di Modena e Reggio Emilia, Modena, Italy.

Corresponding author: S. Orlandini, Dipartimento di Ingegneria "Enzo Ferrari," Università degli Studi di Modena e Reggio Emilia, Via Vignolese 905, IT-41125 Modena, Italy. (stefano.orlandini@unimore.it)

(C)2013. American Geophysical Union. All Rights Reserved. 0043-1397/14/10.1002/2013WR014606
}

reveals that the dispersion produced by multiple flow direction methods strongly depends on grid cell size, and suggests therefore that caution must indeed be exercised in the application of multiple flow direction methods for the description of overland flow dispersion. Even under a broad perspective, slope lines remain essential topographic attributes for the description of overland flows because (1) they provide the surface flow paths along which gravity-driven, nondispersive flows of water and sediments extend, and (2) they provide the skeleton around which dispersive overland flow patterns are likely to develop.

[3] Slope lines and related flow nets can be determined directly from contour elevation data as shown by Moretti and Orlandini [2008]. However, a classical flow net is not suited to natural landscapes because flow strips continually merge or split, and the flow net has to be modified by amalgamating excessively small elements and subdividing excessively large elements [Moore and Grayson, 1991]. Hence, the determination of slope lines in grid digital elevation models remains a relevant problem. This problem is commonly addressed by connecting cell centers along single slope directions obtained from O'Callaghan and Mark's [1984] D8 method. Several methods have been proposed in the literature to mitigate the artifacts produced by the D8 method while still using the minimum amount of dispersion [e.g., Fairfield and Leymarie, 1991; Tarboton, 1997; Orlandini et al., 2003; Paik, 2008; Orlandini and Moretti, 2009a]. Reviews of these methods can be found in Tarboton [1997] and Gallant and Hutchinson [2011]. Other algorithms based on the aspect driven method are not 
considered in the present investigation [e.g., Lea, 1992; Costa-Cabral and Burges, 1994; Zhou et al., 2011]. The D8-LTD (eight slope directions, least transverse deviation) method introduced by Orlandini et al. [2003] was shown numerically to improve the standard D8 method while also preserving important qualities of the D8 method such as being deterministic, nondispersive, robust, and computationally efficient [Orlandini and Moretti, 2009b]. However, as pointed out by Paik [2008], an analytical basis of the D8-LTD method having general validity has not been provided so far.

[4] In the present study, an analytical basis for determining slope lines in grid digital elevation models is provided by combining the D8-LTD method with methods of algebra and calculus. This analytical basis makes it possible to verify the capabilities of the D8-LTD method to approximate consistently the exact slope lines of any sloping surface as the grid cell size goes to zero. The D8-LTD method is described in section 2. The analytical basis of the D8-LTD method is provided in section 3. The capabilities of the D8LTD method over other nondispersive methods are demonstrated numerically in section 4 . The numerical analysis reported in section 4 is extended by evaluating the capabilities of dispersive methods to provide simulated propagation patterns that develop around the exact slope lines. Discussion of results and conclusions are reported in sections 5 and 6 , respectively.

\section{The D8-LTD Method}

\subsection{Steepest Slope Directions}

[5] The elementary computational system sketched in Figure 1a is used to compute the steepest (downward) slope direction for all cells of a grid-based digital elevation model [Tarboton, 1997]. Square cells having size equal to $h$ are considered and a block-centered scheme with each elevation value taken to represent the elevation of the center (or the average elevation) of the corresponding cell is adopted. Eight planar triangular facets are formed between the center of the cell and the centers of the eight neighboring cells. The three-dimensional geometry of each facet is characterized by the elevations $e_{i}(i=0,1,2)$. These elevations are arranged so that $e_{0}$ is in the center point, $e_{1}$ is in the point to the side, and $e_{2}$ is in the diagonal point. For a generic triangular facet, the slope (downward) can be represented by the vector $\left(s_{1}, s_{2}\right)$, where $s_{1}=\left(e_{0}-e_{1}\right) / h$ and $s_{2}=\left(e_{1}-e_{2}\right) / h$. The direction (angle from the cardinal edge of the facet) of the steepest slope line in the facet is computed as $\alpha=\arctan \left(s_{2} / s_{1}\right)$ for $s_{1} \neq 0, \alpha=+\pi / 2 \operatorname{rad}$ for $s_{1}=$ 0 and $s_{2}>0, \alpha=-\pi / 2$ rad for $s_{1}=0$ and $s_{2}<0$, or is conventionally set equal to zero for $s_{1}=0$ and $s_{2}=0$. The related slope magnitude is computed as $s_{\max }=\left(s_{1}^{2}+s_{2}^{2}\right)^{1 / 2}$. If $\alpha$ is not in the angle range of the facet at the center point $[0, \pi / 4 \mathrm{rad}]$, then $\alpha$ needs to be set as the direction along the appropriate edge and $s_{\max }$ assigned as the slope along the edge. If $\alpha<0$, then $\alpha$ and $s_{\max }$ are set equal to 0 and $s_{1}$, respectively. If $\alpha>\pi / 4 \mathrm{rad}$, then $\alpha$ and $s_{\max }$ are set equal to $\pi / 4 \mathrm{rad}$ and $\left(e_{0}-e_{2}\right) /(\sqrt{2} h)$, respectively. Table 1 gives the node elevations corresponding to the corners of each of the triangular facets used to compute slopes and angles. The steepest slope direction associated with a grid cell is determined in the direction of the steepest (downward)
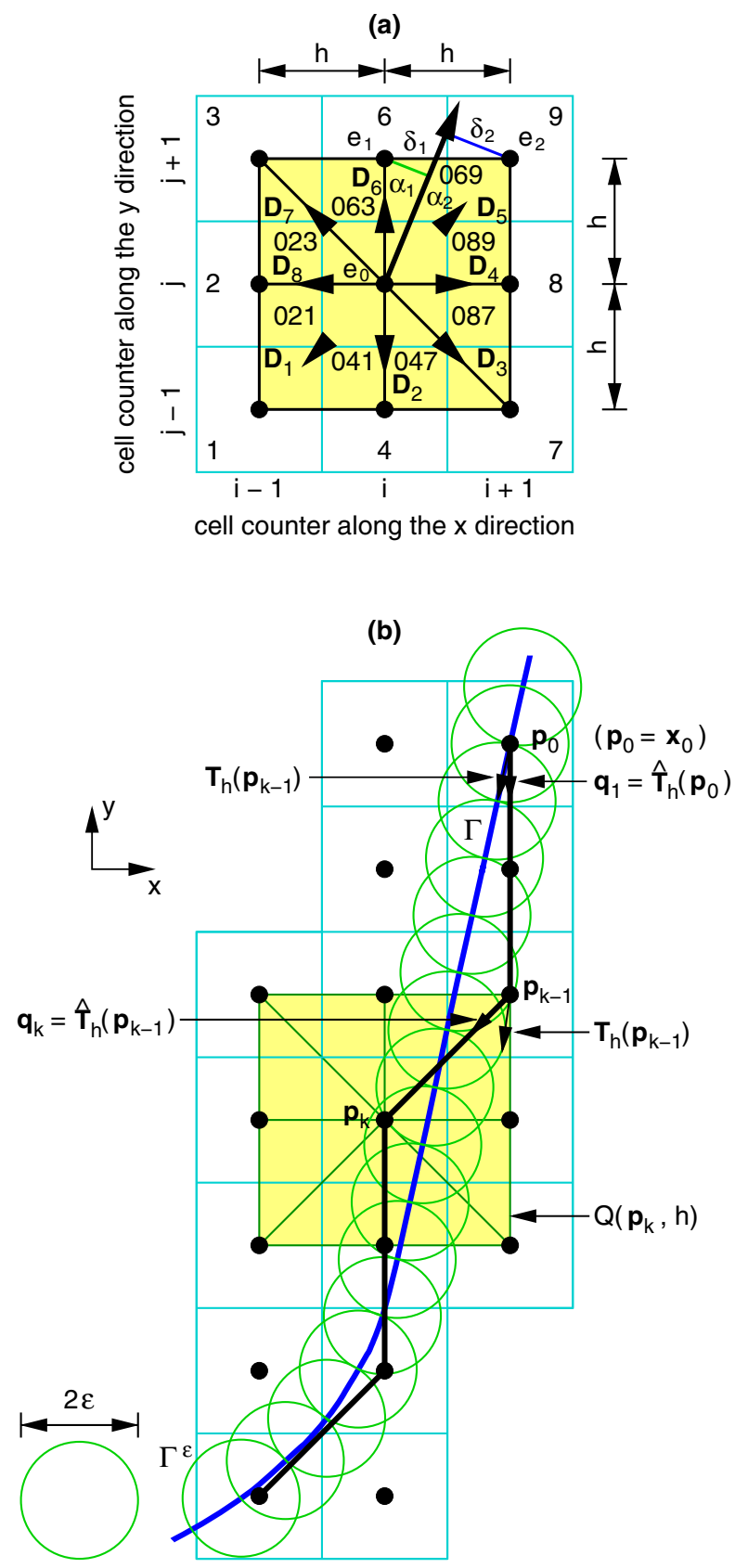

Figure 1. Sketches of (a) the elementary computational systems used to formulate numerically the D8-LTD method and (b) the concepts used to provide an analytical basis for this method.

slope on the eight triangular facets centered on that cell. Equalities are resolved by selecting (among the equal possibilities) the steepest flow directions along the facet that is processed first, starting conventionally from facet 021 in Figure 1a and proceeding in a clockwise manner.

\subsection{Least Angular and Transverse Deviations}

[6] The steepest slope direction computed at a grid cell can vary continuously as an angle between 0 and $2 \pi$ rad and does not generally follow one of the cardinal $(0$, $\pi / 2, \pi$, and $3 \pi / 2 \mathrm{rad})$ or diagonal $(\pi / 4,3 \pi / 4,5 \pi / 4$, and $7 \pi / 4 \mathrm{rad})$ directions that can be selected. Possible slope 
Table 1. Values Taken by the Variables Involved in the D8-LTD Slope Direction Method

\begin{tabular}{|c|c|c|c|c|c|c|c|c|}
\hline \multirow[b]{2}{*}{ Variable } & \multicolumn{8}{|c|}{ Facet } \\
\hline & 21 & 23 & 063 & 069 & 089 & 087 & 047 & 041 \\
\hline$e_{1}$ & $z_{i-1, j}$ & $z_{i-1, j}$ & $z_{i, j+1}$ & $z_{i, j+1}$ & $z_{i+1, j}$ & $z_{i+1, j}$ & $z_{i, j-1}$ & $z_{i, j-1}$ \\
\hline$e_{2}$ & $z_{i-1, j-1}$ & $z_{i-1, j+1}$ & $z_{i-1, j+1}$ & $z_{i+1, j+1}$ & $z_{i+1, j+1}$ & $z_{i+1, j-1}$ & $z_{i+1, j-1}$ & $z_{i-1, j-1}$ \\
\hline$d_{1}$ & 2 & 2 & 6 & 6 & 8 & 8 & 4 & 4 \\
\hline$d_{2}$ & 1 & 3 & 3 & 9 & 9 & 7 & 7 & 1 \\
\hline
\end{tabular}

directions from a grid cell are identified by a pointer $d$ indicating the local cell number of the draining cell. The pointers associated to the cardinal and diagonal directions of the facet containing the steepest slope direction, denoted as $d_{1}$ and $d_{2}$, respectively, are reported in Table 1 . The choice between $d_{1}$ and $d_{2}$ in order to approximate the steepest slope direction with a single slope direction can be made by computing the angular deviations $\alpha_{1}=\alpha$ and $\alpha_{2}=\pi /$ $4 \mathrm{rad}-\alpha$ shown in Figure 1a and by ensuring the least angular deviation (LAD). The direction $d_{1}$ is chosen if $\alpha_{1} \leq \alpha_{2}$, whereas the direction $d_{2}$ is chosen if $\alpha_{1}>\alpha_{2}$. Alternatively, this choice can be made by computing the transverse deviations $\delta_{1}=h \sin \alpha_{1}$ and $\delta_{2}=\sqrt{2} h \sin \alpha_{2}$ shown in Figure 1a and by ensuring the least transverse deviation (LTD). The direction $d_{1}$ is chosen if $\delta_{1} \leq \delta_{2}$, whereas the direction $d_{2}$ is chosen if $\delta_{1}>\delta_{2}$. The LAD criterion is exactly equivalent to selecting the steepest direction among the eight possible direction as made by the classical D8 method. The LTD criterion was introduced to ensure an accurate control of the cumulative deviation along a slope line so as to provide a path-based analysis.

\subsection{Path-Based Analysis}

[7] A path-based analysis of a slope line is performed by accumulating (transverse) deviations along the slope line approximation. A sign $\sigma$ is assigned to each deviation that may occur in the eight triangular facets of the elementary computational system as reported in Table 1 . With the values of $\sigma$ reported in Table 1, an observer looking in the direction of the steepest (downward) slope finds the positive transverse deviations on his right and the negative transverse deviations on his left. At the first cell along a path $(k=1)$, the local (transverse) deviations associated to $d_{1}$ and $d_{2}$ are $\delta_{1}(1)=\delta_{1}$ and $\delta_{2}(1)=\delta_{2}$, respectively. The corresponding cumulative (transverse) deviations are $\delta_{1}^{+}(1)$ $=\sigma \delta_{1}(1)$ and $\delta_{2}^{+}(1)=-\sigma \delta_{2}(1)$. The slope direction from the first cell along the path $(k=1)$ and the cumulative (transverse) deviation $\delta^{+}(1)$ conveyed to the downslope cell are determined as follows:

$$
\begin{aligned}
& \text { If }\left|\delta_{1}^{+}(1)\right| \leq\left|\delta_{2}^{+}(1)\right| \text {, then } d=d_{1}, \delta^{+}(1)=\delta_{1}^{+}(1) \\
& \text { If }\left|\delta_{1}^{+}(1)\right|>\left|\delta_{2}^{+}(1)\right| \text {, then } d=d_{2}, \delta^{+}(1)=\delta_{2}^{+}(1) \text {. }
\end{aligned}
$$

[8] On subsequent cells along the path $(k=2,3, \cdots)$, the local (transverse) deviations associated to $d_{1}$ and $d_{2}$ are $\delta_{1}(k)=\delta_{1}$ and $\delta_{2}(k)=\delta_{2}$, respectively. The corresponding cumulative (transverse) deviations are computed as

$$
\delta_{1}^{+}(k)=\sigma \delta_{1}(k)+\delta^{+}(k-1)
$$

and

$$
\delta_{2}^{+}(k)=-\sigma \delta_{2}(k)+\delta^{+}(k-1),
$$

where $\delta^{+}(k-1)$ is the cumulative (transverse) deviation conveyed to the $k$ th cell along the path. The slope direction from the $k$ th cell along the path $(k=2,3, \cdots)$ and the cumulative (transverse) deviation $\delta^{+}(k)$ conveyed to the downslope cell are determined as follows:

$$
\begin{aligned}
& \text { If }\left|\delta_{1}^{+}(k)\right| \leq\left|\delta_{2}^{+}(k)\right| \text {, then } d=d_{1}, \delta^{+}(k)=\delta_{1}^{+}(k) . \\
& \text { If }\left|\delta_{1}^{+}(k)\right|>\left|\delta_{2}^{+}(k)\right| \text {, then } d=d_{2}, \delta^{+}(k)=\delta_{2}^{+}(k) .
\end{aligned}
$$

[9] The term $\delta^{+}(k-1)$ in equations (3) and (4) is given by (1) and (2) for $k=2$, or by (5) and (6) for $k>2$. The path-based analysis described in the present section is denoted as D8-LTD method when the LTD criterion (section 2.2) is used, or D8-LAD method when the LAD criterion (section 2.2) is used [Orlandini et al., 2003]. It is highlighted here that no boundary conditions nor parameters need to be specified in the D8-LTD and D8-LAD methods. The fundamental case of a single slope line beginning at a point is considered in the present study. Slope lines that join to form a network can be handled as reported in Orlandini and Moretti [2009b]. Clearly, an accurate description of a slope line network is obtained by describing accurately each single slope line composing this network.

\section{Analytical Basis of the D8-LTD Method}

[10] In the present section, an analytical basis for the D8LTD method is provided by introducing suitable mathematical notations. Methods of algebra and calculus are applied to a generic $C^{2}$ function with nonzero gradient representing a sloping surface in order to obtain results having general validity. It is shown that slope lines determined in grid digital elevation models by using the D8-LTD method can approximate the exact slope lines as close as desired by selecting a grid cell size that is small enough.

[11] Let land surface topography be represented by a sufficiently smooth (in the sense specified below) function $z=$ $z(\mathbf{x})$ describing the variation of land surface elevation $z$ as a function of points whose planar position vector is $\mathbf{x}=(x, y)$. The land surface gradient is denoted by $\nabla z=\left(z_{x}^{\prime}, z_{y}^{\prime}\right)$, where $z_{x}^{\prime}$ and $z_{y}^{\prime}$ denote the derivatives of $z$ with respect to $x$ and $y$, 
respectively. A slope line $\Gamma$ beginning at the initial point $\mathbf{x}_{0}$ $=\left(x_{0}, y_{0}\right)$ is denoted as

$$
\Gamma=\left\{\mathbf{x}(t) ; t \geq t_{0}\right\}
$$

where $t$ is an independent variable and the function $\mathbf{x}(t)$ is the solution of the initial value problem given by the differential equation

$$
\mathbf{x}^{\prime}(t)=-\nabla z(\mathbf{x}(t))
$$

where $\mathbf{x}^{\prime}(t)$ is the derivative of $\mathbf{x}$ with respect to $t$, together with the specified initial condition

$$
\mathbf{x}\left(t_{0}\right)=\mathbf{x}_{0} .
$$

[12] To ensure that the solution of the initial value (Cauchy) problem given by equations (8) and (9) is unique, it is required that $z$ is a $C^{2}$ function (i.e., a function that has continuous partial derivatives up to the second order). This requirement does not affect the generality of the analysis presented because any complex topography or microtopography can, at least in principle, be represented by a $C^{2}$ function that is detailed enough. It is also required that $\nabla z \neq 0$ everywhere, so that the solution $\mathbf{x}(t)$ never stops. This requirement is consistent with the use of grid digital elevation models displaying no sinks (i.e., cells having no neighbors at a lower elevation). Such digital elevation models are obtained from raw digital elevation models by applying, if necessary, algorithms that identify and treat all flat areas and close depressions [e.g., Martz and Garbrecht, 1992; Grimaldi et al., 2007]. It is finally assumed that $\Gamma$ lies entirely within the considered domain and is therefore not constrained by boundary conditions. Under this assumption, $z$ can be thought to be defined on the whole plane $\mathbb{R}^{2}$, where $\mathbb{R}$ denote the set of real numbers, without loss of generality.

[13] Let $G(h)=\{(i h, j h) ; i, j \in \mathbb{Z}\}$ denote the set of cell centers in which the elevations $z$ of the grid digital elevation model are provided, where $h$ is the grid cell size, $i$ and $j$ are counters belonging to the set of integer numbers $\mathbb{Z}$ (section 2, Figure 1b). In our coordinate system, $G(h)$ is made up by points $\mathbf{x}=(x, y)$ such that both $x$ and $y$ are integer multiples of $h$. As shown in Figure $1 \mathrm{~b}$, for any point $\mathbf{p}$ $\in G(h)\left(\mathbf{p}=\mathbf{p}_{k}\right.$ in Figure $\left.1 \mathrm{~b}\right)$, the notation $Q(\mathbf{p} ; h)$ denotes the square having center in $\mathbf{p}$ and size $2 h$. In accordance to the scheme shown in Figure $1 \mathrm{a}, Q(\mathbf{p} ; h)$ is divided into eight triangles, with a common vertex at $\mathbf{p}$. The exact slope direction we should follow at $\mathbf{p}$ is given by the unit vector

$$
\mathbf{T}(\mathbf{p})=-\nabla z(\mathbf{p}) /\|\nabla z(\mathbf{p})\| .
$$

[14] When a grid digital elevation model is used as reported in section 2, $z$ is only known at the points of $G(h)$. Then we take, instead of $\mathbf{T}(\mathbf{p})$, a vector $\mathbf{T}_{h}(\mathbf{p})$ which minimizes, among all possible unit vectors $\boldsymbol{v}$, the value $\Delta_{h}(\mathbf{p} ; \boldsymbol{v})$ of the one-sided directional derivative at $\mathbf{p}$, along $\boldsymbol{v}$, of the function which agrees with $z$ on the vertices of the eight triangles, and is linear on each of these triangles. Then, by denoting as $\mathbf{v}_{h}^{i}(\mathbf{p})$ the gradient of this function on the $i$ th triangle, for any $h>0$ there is at least one index $i(h)$ $\in\{1, \cdots, 8\}$ such that

$$
\Delta_{h}\left(\mathbf{p} ; T_{h}(\mathbf{p})\right)=\mathbf{v}_{h}^{i(h)}(\mathbf{p}) \cdot \mathbf{T}_{h}(\mathbf{p}) \leq \Delta_{h}(\mathbf{p} ; \boldsymbol{v}), \quad \boldsymbol{v} \in S,
$$

where - denotes the (dot) scalar product of two vectors, while $S$ stands for the set of all unit vectors of the plane. Since, however, we can only move along the eight admissible vectors $\mathbf{D}_{1}, \cdots, \mathbf{D}_{8}$, we replace in turn $\mathbf{T}_{h}(\mathbf{p})$ by one of these vectors, say $\hat{\mathbf{T}}_{h}(\mathbf{p})$, whose norm can either be equal to 1 or $\sqrt{2}$ depending on whether a cardinal or a diagonal direction is selected. The choice of $\hat{\mathbf{T}}_{h}(\mathbf{p})$ is performed according to the procedure we explained in section 2 . In the present section, however, the scripts $\delta_{i}(k, h)(i=1,2)$ and $\delta^{+}(k, h)$ are adopted in preference to $\delta_{i}(k)(i=1,2)$ and $\delta^{+}(k)$ in order to put in evidence the dependence on the parameter $h$. As sketched in Figure 1b, starting from a given point $\mathbf{p}_{0}$ the discrete vector field $\hat{\mathbf{T}}_{h}$ determines a sequence of points $\mathbf{p}_{0}(h), \mathbf{p}_{1}(h), \mathbf{p}_{2}(h), \cdots$ in $G(h)$, which are defined by recurrence as

$$
\begin{gathered}
\mathbf{p}_{0}(h)=\mathbf{p}_{0}, \\
\mathbf{p}_{k}(h)=\mathbf{p}_{k-1}(h)+h \mathbf{q}_{k}(h), \quad k=1,2, \cdots,
\end{gathered}
$$

where

$$
\mathbf{q}_{k}(h)=\hat{\mathbf{T}}_{h}\left(\mathbf{p}_{k-1}(h)\right) .
$$

[15] In particular, the transverse deviation $\delta(k, h)$ of $h$ $\mathbf{q}_{k}(h)$ with respect to the direction given by the vector $\mathbf{T}_{h}\left(\mathbf{p}_{k-1}(h)\right)$ is expressed by $\delta(k, h)=|\tilde{\delta}(k, h)|$, where

$$
\tilde{\delta}(k, h)=h \mathbf{q}_{k}(h) \cdot \mathbf{A} \mathbf{T}_{h}\left(\mathbf{p}_{k-1}(h)\right)
$$

[16] In equation (15), $\mathbf{A}$ is the clockwise rotation matrix by the angle $\pi / 2 \mathrm{rad}$. It can be noted that $\tilde{\delta}(k, h)=\sigma \delta(k, h)$, where $\sigma$ is the sign which appears in equations (3) and (4). We also note that $\tilde{\delta}(k, h)$ is the determinant of the $2 \times 2$ matrix whose columns are the vectors $h \mathbf{q}_{k}(h)$ and $\mathbf{T}_{h}\left(\mathbf{p}_{k-1}(h)\right)$. Since $\left\|\mathbf{T}_{h}\left(\mathbf{p}_{k-1}(h)\right)\right\|=1, \tilde{\delta}(k, h)$ is the product between the norm of $h \mathbf{q}_{k}(h)$ with the sine of the oriented angle $\alpha$ between the two vectors. The norm of $h \mathbf{q}_{k}(h)$ is equal to $\sqrt{2} h$ for $\mathbf{D}=\mathbf{D}_{1}, \mathbf{D}_{3}, \mathbf{D}_{5}, \mathbf{D}_{7}$ (diagonal directions), or rather equal to $h$ for $\mathbf{D}=\mathbf{D}_{2}, \mathbf{D}_{4}, \mathbf{D}_{6}, \mathbf{D}_{8}$ (cardinal directions). We also recall that $\hat{\mathbf{T}}_{h}\left(\mathbf{p}_{k-1}(h)\right)$ is chosen between two vectors of the finite set $\left\{\mathbf{D}_{1}, \cdots, \mathbf{D}_{8}\right\}$, which we denote, respectively, by $\mathbf{q}_{k}^{1}(h)$ and $\mathbf{q}_{k}^{2}(h)$. Accordingly, the transverse deviation $\delta(k, h)$ is one of the two numbers $\mid \tilde{\delta}_{1}($ $k, h) \mid$ and $\left|\tilde{\delta}_{2}(k, h)\right|$ which we get on replacing $\mathbf{q}_{k}(h)$ in equation (15) by $\mathbf{q}_{k}^{1}(h)$ and $\mathbf{q}_{k}^{2}(h)$, respectively. Now, since $-\pi / 4 \leq \alpha \leq \pi / 4 \mathrm{rad}$, one obtains that

$$
-h \leq \tilde{\delta}_{1}(k, h) \leq 0 \leq \tilde{\delta}_{2}(k, h) \leq h
$$

and

$$
h / \sqrt{2} \leq \eta(k, h) \leq \sqrt{2} h,
$$

where $\eta(k, h)=h \mathbf{q}_{k}(h) \cdot \mathbf{T}_{h}\left(\mathbf{p}_{k-1}(h)\right)$ is the component of $h$ $\mathbf{q}_{k}(h)$ along the tangent to the slope line.

[17] With the preliminary remarks reported above, the central question of the present investigation is: what happens to the sequence $\mathbf{p}_{0}(h), \mathbf{p}_{1}(h), \mathbf{p}_{2}(h), \cdots$ (equations $(12-14))$ as the size $h$ of the cells converges to zero? To 
answer this question, let us fix a number $r>0$ and consider, for any $n \in \mathbb{Z}^{+}$, the polygonal path $\Gamma_{n}$ whose vertices are the $n+1$ points

$$
\mathbf{p}_{0}, \mathbf{p}_{1}(r / n), \mathbf{p}_{2}(r / n), \cdots, \mathbf{p}_{n}(r / n)
$$

according to the notation introduced in equation (13). When $n$ diverges to $+\infty$, the size $h_{n}=r / n$ of the cells converges to zero. Our aim is to show that $\Gamma_{n}$ approaches $\Gamma$ in a sense that we are going to explain. To this end, for any $\varepsilon>0$, we introduce the $\varepsilon$-neighborhood of the slope line $\Gamma$ in equation (7), namely

$$
\Gamma^{\varepsilon}=\bigcup_{\mathbf{x} \in \Gamma} B(\mathbf{x} ; \varepsilon),
$$

where $B(\mathbf{x} ; \varepsilon)$ stands for the disk with center $\mathbf{x}$ and radius $\varepsilon$ (Figure 1b). Now we are able to state our approximation result in a rigorous form.

[18] Theorem 3.1. Let $z: \mathbb{R}^{2} \rightarrow \mathbb{R}$ be a $C^{2}$-function whose gradient $\nabla z$ never vanishes. Let $\mathbf{p}_{0} \in \mathbb{R}^{2}$ be given, and $\mathbf{x}(t)$ be the solution of equations (8) and (9) with $\mathbf{x}_{0}=\mathbf{p}_{0}$. Let us recall the definition of $\Gamma, \Gamma_{n}$, and $\Gamma^{\varepsilon}$ in equations (7), (18), and (19). Then, for any $\varepsilon>0$, there exists $n_{\varepsilon}$ $\in \mathbb{Z}^{+}$such that

$$
\Gamma_{n} \subseteq \Gamma^{\varepsilon}, \quad n \geq n_{\varepsilon} .
$$

[19] In order to prove Theorem 3.1, we need some preliminary results. First of all, we point out that our assumptions on $z$ allow us to find, whenever $\mathbf{x}$ ranges over a bounded region of the plane, three constants $m, M$, and $K$ such that

$$
\begin{gathered}
0<m \leq\|\nabla z(\mathbf{x})\| \leq M \\
\left\|\nabla^{2} z(\mathbf{x})\right\|=\left(z_{x x}^{\prime \prime}(\mathbf{x})^{2}+2 z_{x y}^{\prime \prime}(\mathbf{x})^{2}+z_{y y}^{\prime \prime}(\mathbf{x})^{2}\right)^{1 / 2} \leq K .
\end{gathered}
$$

[20] Actually, since we are always working in a region which can be bounded a priori (such as, for instance, a drainage basin), it is right to suppose that $m, M$, and $K$ are fixed. Furthermore, we point out that we are interested in $\Gamma$ as a set of points, not in the way $\mathbf{x}(t)$ runs over it. From this point of view, the differential equation in equation (8) can be replaced by more general conditions. Roughly speaking, the important feature of the vector at the right-hand side of equation (8) is not the norm, but rather the oriented direction. We are going to state this property in a more precise form, through the next result.

[21] Lemma 3.2. Let $\mathbf{p}_{0} \in \mathbb{R}^{2}, r>0, \hat{\mathbf{x}}:[0, r] \rightarrow \mathbb{R}^{2}$ be a Lipschitz continuous function [e.g., Vulikh, 1963, p. 109] such that $\hat{\mathbf{x}}(0)=\mathbf{p}_{0}$ and, for almost every $t \in[0, r]$ :

$$
\begin{gathered}
\hat{\mathbf{x}}^{\prime}(t) \| \nabla z(\hat{\mathbf{x}}(t)), \\
\hat{\mathbf{x}}^{\prime}(t) \cdot \nabla z(\hat{\mathbf{x}}(t)) \leq-c,
\end{gathered}
$$

where the symbol \| in equation (23) means "parallel to" and $c>0$ is given. Then, $\hat{\mathbf{x}}(t) \in \Gamma$ for any $t \in[0, r]$.

[22] The proof of Lemma 3.2 is reported in Appendix A.

[23] We recall that, according to our previous notations, the cumulative deviation at the $k$ th step can be written as $\delta^{+}(k, h)=\sum_{j=1}^{k} \tilde{\delta}(j, h)$.
[24] Lemma 3.3. $\left|\delta^{+}(k, h)\right| \leq h$.

[25] Proof of Lemma 3.3. Let us fix $h>0$ and, for notational convenience, let us drop the dependence on $h$ in the scripts $\tilde{\delta}(k, h), \delta^{+}(k, h), \tilde{\delta}_{i}(k, h)(i=1,2)$. We recall that, according to the D8-LTD method (section 2.3):

$$
\text { If }\left|\delta^{+}(k-1)+\tilde{\delta}_{2}(k)\right| \leq\left|\delta^{+}(k-1)+\tilde{\delta}_{1}(k)\right|, \quad \text { then } \tilde{\delta}(k)=\tilde{\delta}_{2}(k)
$$

$$
\text { If }\left|\delta^{+}(k-1)+\tilde{\delta}_{2}(k)\right|>\left|\delta^{+}(k-1)+\tilde{\delta}_{1}(k)\right|, \quad \text { then } \tilde{\delta}(k)=\tilde{\delta}_{1}(k)
$$

[26] Now, since our claim obviously holds for $k=1$, let us argue by induction and suppose that $\left|\delta^{+}(k-1)\right| \leq h$. In order to fix ideas, let $\delta^{+}(k-1) \geq 0$. Then, according also to equation (16):

$$
\begin{gathered}
-h \leq \delta_{1}(k) \leq \delta^{+}(k-1)+\delta_{1}(k) \leq \delta^{+}(k-1) \leq h, \\
\left|\delta^{+}(k)\right|=\left|\delta^{+}(k-1)+\delta(k)\right| \leq\left|\delta^{+}(k-1)+\delta_{1}(k)\right| \leq h,
\end{gathered}
$$

where the last inequality of equation (28) follows from equation (27). In the same way, we can proceed if $\delta^{+}(k-1)$ $\leq 0$ so as to complete the proof.

[27] Now, in order to parameterize $\Gamma_{n}$, let us divide $[0, r]$ into $n$ intervals $J(n, k)$, where $J(n, k)=[r(k-1) / n, r k / n)$ for $k=1, \cdots, n-1$, and $J(n, n)=[r(n-1) / n, r]$. Now, define a map $\mathbf{x}_{n}:[0, r] \rightarrow \mathbb{R}^{2}$ by putting $\mathbf{x}_{n}(t)=\mathbf{p}_{k-1}(r / n)+$ $(t-r(k-1) / n) \mathbf{q}_{k}(r / n)$, where $t \in J(n, k)$. Note that $\mathbf{x}_{n}$ is the piecewise linear function on $[0, r]$ which takes the values $\mathbf{p}_{k}(r / n)$ at the points $k r / n$, where $k=0,1, \cdots, n$. In particular: $\quad \mathbf{x}_{n}^{\prime}(t)=\mathbf{q}_{k}(r / n), \quad$ whenever $\quad t \in J(n, k)$, $t \neq r(k-1) / n$. Hence, the following evaluations hold:

$$
\left\|\mathbf{x}_{n}^{\prime}(t)\right\| \leq \sqrt{2}, \quad\left\|\mathbf{x}_{n}(t)\right\| \leq\left\|\mathbf{p}_{0}\right\|+r \sqrt{2} .
$$

[28] From equation (29) and the Arzelà-Ascoli theorem [e.g., Taylor and Lay, 1980, p. 295], we argue that $\mathbf{x}_{n}$ converges uniformly to a continuous function $\hat{\mathbf{x}}$ along a suitable sequence of indexes $n \in \mathbb{Z}^{+}$. In order to simplify the exposition we suppose, at least for the moment, that the whole sequence converges uniformly on $[0, r]$ :

$$
\mathbf{x}_{n} \rightarrow \hat{\mathbf{x}} \quad \text { as } n \rightarrow+\infty
$$

[29] Toward the end of the section, we shall explain how to remove this additional assumption. From equations (30) and (29), we also infer what follows: $\hat{\mathbf{x}}$ is Lipschitz continuous and, for any continuous function $\mathbf{v}:[0, r] \rightarrow \mathbb{R}^{2}$ and any $s, t \in[0, r]$ :

$$
\int_{s}^{t} \mathbf{x}_{n}^{\prime}(\tau) \cdot \mathbf{v}(\tau) d \tau \rightarrow \int_{s}^{t} \hat{\mathbf{x}}^{\prime}(\tau) \cdot \mathbf{v}(\tau) d \tau \quad \text { as } n \rightarrow+\infty
$$

[30] The proof of this assertion can be provided by using well-known results on weak compactness in functional spaces [e.g., Brezis, 2010, p. 55]. Now, let us define a step function $\hat{\mathbf{q}}_{n}:[0, r] \rightarrow \mathbb{R}^{2}$ as $\hat{\mathbf{q}}_{n}(t)=\mathbf{T}_{r / n}\left(\mathbf{p}_{k-1}(r / n)\right)$ for $t \in J(n, k)$, where $\mathbf{T}_{h}$ is defined by equation (11). Then, one can check that 


$$
\int_{0}^{r k / n} \mathbf{x}_{n}^{\prime}(\tau) \cdot \mathbf{A} \hat{\mathbf{q}}_{n}(\tau) d \tau=\delta^{+}(k, r / n)
$$

[31] In particular, by virtue of Lemma 3.3:

$$
\int_{0}^{r k_{n} / n} \mathbf{x}_{n}^{\prime}(\tau) \cdot \mathbf{A} \hat{\mathbf{q}}_{n}(\tau) d \tau \rightarrow 0 \quad \text { as } n \rightarrow+\infty
$$

whenever the integers $k_{n}$ are chosen between 1 and $n$. Since any point $t \in[0, r]$ can be approximated by points of the kind $r k_{n} / n$, from (33) we argue that actually, for any $t \in[0, r]$,

$$
\int_{0}^{t} \mathbf{x}_{n}^{\prime}(\tau) \cdot \mathbf{A} \hat{\mathbf{q}}_{n}(\tau) d \tau \rightarrow 0 \quad \text { as } n \rightarrow+\infty
$$

[32] As we already explained, $\mathbf{T}_{h}$ can be regarded as a discrete approximation, on the grid $G(h)$, of the field $\mathbf{T}$ of equation (10). In order to be more precise, let us recall the constant $K$ from equation (22) and the vectors $\mathbf{v}_{h}^{i}(\mathbf{p})$ which were defined before equation (11). Then some technical computations yield the following estimate, which will be useful in the next result:

$$
\left\|\mathbf{v}_{h}^{i}(\mathbf{p})-\nabla z(\mathbf{p})\right\| \leq 4 K h, \quad h>0, \quad \mathbf{p} \in \mathbb{R}^{2}, \quad i=1, \cdots, 8 .
$$

[33] Lemma 3.4. Let us put $h^{*}=m /(8 K)$, $C=6 \sqrt{2 K / m}$, where $m$ and $K$ come from equations (21) and (22). Then $\left\|\mathbf{T}_{h}(\mathbf{p})-\mathbf{T}(\mathbf{p})\right\| \leq C \sqrt{h}$ for any $h \in\left(0, h^{*}\right], \mathbf{p} \in \mathbb{R}^{2}$.

[34] Lemma 3.5. For any $n \in \mathbb{Z}^{+}, t \in[0, r]$ let us put $\hat{\mathbf{q}}(t)=\mathbf{T}(\hat{\mathbf{x}}(t))$. Then $\hat{\mathbf{q}}_{n} \rightarrow \hat{\mathbf{q}}$ as $n \rightarrow+\infty$ uniformly on $[0, r]$.

[35] Lemma 3.6. The function $\hat{\mathbf{x}}$ fulfills equations (23) and (24).

[36] The proofs of the previous three lemmas are postponed to Appendices B, C, and D, respectively.

[37] Proof of Theorem 3.1. Let us first assume that the whole sequence $\mathbf{x}_{n}$ satisfies equation (30), fix $\varepsilon>0$, and take $n_{\varepsilon} \in \mathbb{Z}^{+} \quad$ such that $\left\|\mathbf{x}_{n}(t)-\hat{\mathbf{x}}(t)\right\| \leq \varepsilon$ for any $n \geq n_{\varepsilon}, t \in[0, r]$. Hence, for such indexes $n$, we get $\Gamma_{n} \subseteq \hat{\Gamma}^{\varepsilon}$, where $\hat{\Gamma}=\hat{\mathbf{x}}([0, r])$ and $\hat{\Gamma}^{\varepsilon}$ is the $\varepsilon$-neighborhood of $\hat{\Gamma}$, which is defined in the same way as in equation (19). On the other hand, by virtue of Lemmas 3.6 and 3.2 the inclusion $\hat{\Gamma} \subseteq \Gamma$ holds as well, and obviously entails $\hat{\Gamma}^{\varepsilon} \subseteq \Gamma^{\varepsilon}$. Then,

$$
\Gamma_{n} \subseteq \hat{\Gamma}^{\varepsilon} \subseteq \Gamma^{\varepsilon}, \quad n \geq n_{\varepsilon}
$$

[38] Now we only need to prove our claim in lack of the additional assumption (30). So, by contradiction, let the thesis of Theorem 3.1 be false. Then there exists $\varepsilon>0$ such that

$$
\Gamma_{n} \not \subset \hat{\Gamma}^{\varepsilon}, \quad n \in H
$$

where $H$ is a suitable, infinite subset of $\mathbb{Z}^{+}$. By virtue of Ascoli-Arzelà theorem, we can now suppose that (30) holds when $n$ ranges over a suitable, infinite subset $H^{\prime}$ of $H$. Now we can argue as before, but on the subsequence $\left\{\mathbf{x}_{n} ; n \in H^{\prime}\right\}$, and infer the following property. For any $\varepsilon>0$, there exists $n_{\varepsilon} \in H^{\prime}$ such that

$$
\Gamma_{n} \subseteq \hat{\Gamma}^{\varepsilon}, \quad n \in H^{\prime}, n \geq n_{\varepsilon} .
$$

[39] Since $H^{\prime} \subseteq H$, (38) is in contrast with (37), so we got the required contradiction.

[40] Remark 3.7. The control on the cumulative transverse deviation $\delta^{+}(k, h)$ (Lemma 3.3) is the key factor for proving that the limit function $\hat{\mathbf{x}}$ fulfills condition (23), since it entails, by virtue of equation (32), the convergence in (34). In lack of such a control, the thesis of Theorem 3.1 could be false.

[41] Remark 3.8. The D8-LAD method (section 2.3) can be examined by applying an analysis similar to the one reported above for the D8-LTD method, in which the quantity $\delta(k, h)$ defined in equation (15) is replaced by an oriented angle, say $\theta(k, h)$, between the two vectors $\mathbf{q}_{k}(h)$ and $\mathbf{T}_{h}\left(\mathbf{p}_{k-1}\right)$. In this different setting, the inductive argument of Lemma 3.3 applies again to the corresponding cumulative angular deviation, say $\delta_{a}^{+}(k, h)$, and shows obviously that its absolute value does not exceed $\pi / 4 \mathrm{rad}$. We note, however, that $\delta_{a}^{+}(k, r / n)$ cannot replace $\delta^{+}(k, r / n)$ in equation (32), and it looks difficult, without such an equality, to estimate properly the integral on the left-hand side to obtain (34).

\section{Numerical Demonstrations}

[42] The capability of the D8-LTD method to predict consistently exact slope lines as the grid cell size goes to zero is demonstrated numerically. In addition, the capabilities of the Tarboton's [1997] D $\infty$, Seibert and McGlynn's [2007] MD $\infty$, and Quinn et al.'s [1991] MD8 dispersive methods to provide simulated propagation patterns that develop around the exact slope lines are evaluated. It should be pointed out here that these dispersive methods are not designed to predict slope lines but rather to describe overland flow patterns affected by dispersion. The analysis of dispersive methods reported in this section is only meant to illustrate how dispersive propagation patterns from point sources develop in relation to the exact slope lines from the same point sources.

\subsection{Test Cases}

[43] Suitable synthetic test cases are obtained by using a modified form of the Himmelblau's [1972] function $Z=\left(X^{2}+Y-11\right)^{2}+\left(Y^{2}+X-7\right)^{2}$, namely

$$
Z=\left(X^{2}+Y-4\right)^{2}+\left(Y^{2}+X-7\right)^{2}
$$

where $Z$ is the vertical coordinate, $X$ and $Y$ are planar coordinates ranging in the intervals $-5 \leq X \leq 5$ and $-5 \leq Y \leq 5$, respectively. In order to obtain a test case representing real topographic systems, the spatial coordinates $X, Y$, and $Z$ are scaled and translated by using the relationships

$$
\begin{gathered}
x=50 X+250, \\
y=50 Y+250, \quad \text { and } \\
z=-0.75 Z+450 .
\end{gathered}
$$

[44] The portion of the surface defined by equations (3942) satisfying $75 \leq x \leq 400 \mathrm{~m}$ and $50 \leq y \leq 450 \mathrm{~m}$ is shown in Figure 2 . For this surface portion 


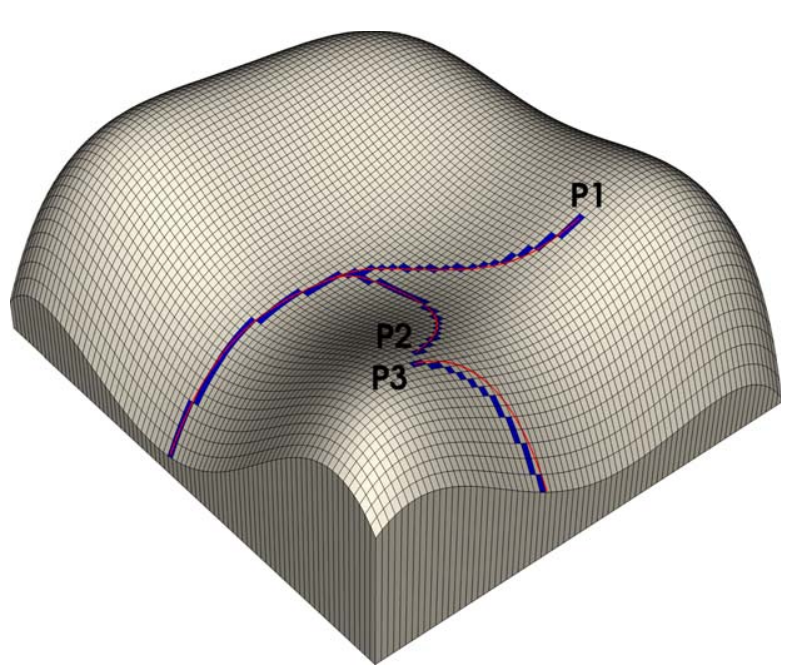

Figure 2. Grid digital elevation model of the modified Himmelblau function (equations (39-42)) used to demonstrate the capabilities of slope direction methods to provide simulated propagation patterns that reproduce or develop around the exact slope lines. Grid cell size is equal to $10 \mathrm{~m}$. Exact slope lines from the initial points P1, P2, and P3 are reported in red. The propagation patterns simulated, for demonstration, by using the D8-LTD method are shown by blue cells.

$300 \leq z \leq 450 \mathrm{~m}$. Other portions of the same surface are considered in Figure $3 \quad(0 \leq x \leq 350 \mathrm{~m} \quad$ and $130 \leq y \leq 280 \mathrm{~m})$ and in Figures 4 and $5(0 \leq x \leq 500 \mathrm{~m}$ and $0 \leq y \leq 500 \mathrm{~m})$. Around the corners of the maps reported in Figures 4 and 5, where the surface is very steep, contour lines are not reported. In the maps reported in Figures 3-5, the intervals between dark gray contour lines are equal to $50 \mathrm{~m}$, and each of these intervals is subdivided if necessary into five subintervals equal to $10 \mathrm{~m}$ by using four light gray contour lines.

[45] Along any slope line defined in the space $X Y$, the slope line element $d \mathbf{S}=(d X, d Y)$ and the gradient $\nabla Z=\left(Z_{x}^{\prime}\right.$, $Z_{y}^{\prime}$ ) must obey the relation $d \mathbf{S} \times \nabla Z=0$, where the symbol $X$ denotes the (cross) vector product, implying that $d Y / d X=-Z_{x}^{\prime} / Z_{y}^{\prime}$. From equation (39), it can therefore be obtained that

$$
\frac{d Y}{d X}=\frac{X^{2}+Y-4+2 Y\left(Y^{2}+X-7\right)}{2 X\left(X^{2}+Y-4\right)+Y^{2}+X-7} .
$$

[46] A slope line beginning at a given point $\left(X_{0}, Y_{0}\right)$ can be delineated by integrating equation (43) from the initial conditions $X=X_{0}$ and $Y=Y_{0}$. Similarly, in the space $x y$ a slope line beginning at a given point $\left(x_{0}, y_{0}\right)$ can be delineated by integrating equation (43) combined with equations (40-42) from the initial conditions $x=x_{0}$ and $y=y_{0}$. In the present study, such an integration is performed numerically by applying an implicit Runge-Kutta formula with a first stage that is a trapezoidal rule step and a second stage that is a backward differentiation formula of order two (ode23tb solver available in Matlab). The obtained slope lines provide suitable benchmark solutions for the evaluations of the D8, D8-LAD, D8-LTD, D $\infty, \mathrm{MD} \infty$, and MD8 methods. It is remarked that these terrain analysis methods are not classical finite difference methods approximating the derivatives of a differential equation with quotients. A differential equation governing the delineation of slope lines is only known for sloping surfaces defined analytically, such as the one considered in the present section (equations (39-43)). The terrain analysis methods considered in the present investigation are rather designed to determine slope lines in grid digital elevation models of any (analytically unknown) sloping surface by using slope line elements connecting cell centers. The test cases reported in Figures 2-5 are obtained by considering the initial points $\mathrm{P} 1, \mathrm{P} 2$, and $\mathrm{P} 3$, the exact slope lines beginning at these points (red lines), and the corresponding simulated propagation patterns resulting from slope direction methods (cells with blue shades).

[47] In Figure 3, simulated propagation patterns from P1 are obtained by applying the D8, D8-LAD, and D8-LTD methods to a $0.5 \mathrm{~m}$ resolution digital elevation model of the surface defined by equations (39-42). It is specified here that the D8 method can be obtained from a local scale application of the LAD criterion (section 2.2), whereas the D8-LAD method is rather obtained from a path-based application the LAD criterion (section 2.3). The simulated propagation patterns from P1 obtained by applying the D8, D8-LAD, and D8-LTD methods in digital elevation models having resolutions of 1,5 , and $10 \mathrm{~m}$ are reported in Figure 4. The simulated propagation patterns from P2 and P3 obtained by applying the D8, D8-LAD, D8-LTD, D $\infty$, $\mathrm{MD} \infty$, and MD8 methods in a $10 \mathrm{~m}$ digital elevation model are reported in Figure 5.

\subsection{Evaluation Metrics}

[48] Two evaluation metrics are used in the present study to quantify the capabilities of slope direction methods to provide simulated propagation patterns that reproduce or develop around the exact slope lines. The first metric is the weighted mean of the deviation distance between the cells of the simulated propagation pattern and the exact slope line. This mean is defined as

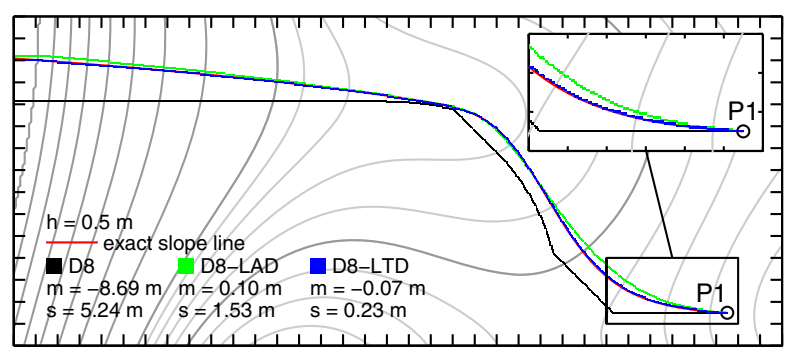

Figure 3. Numerical demonstration of the D8-LTD method propagation pattern (blue cells) capabilities to reproduce the exact slope line (red line) beginning at point P1 when a small grid cell size $(h=0.5 \mathrm{~m})$ is used. These capabilities are not displayed by the D8 method (black cells) or by the D8-LAD method (green cells) propagation patterns. The interval between light gray contour lines is equal to $10 \mathrm{~m}$ and the interval between dark gray contour lines is equal to $50 \mathrm{~m}$. 

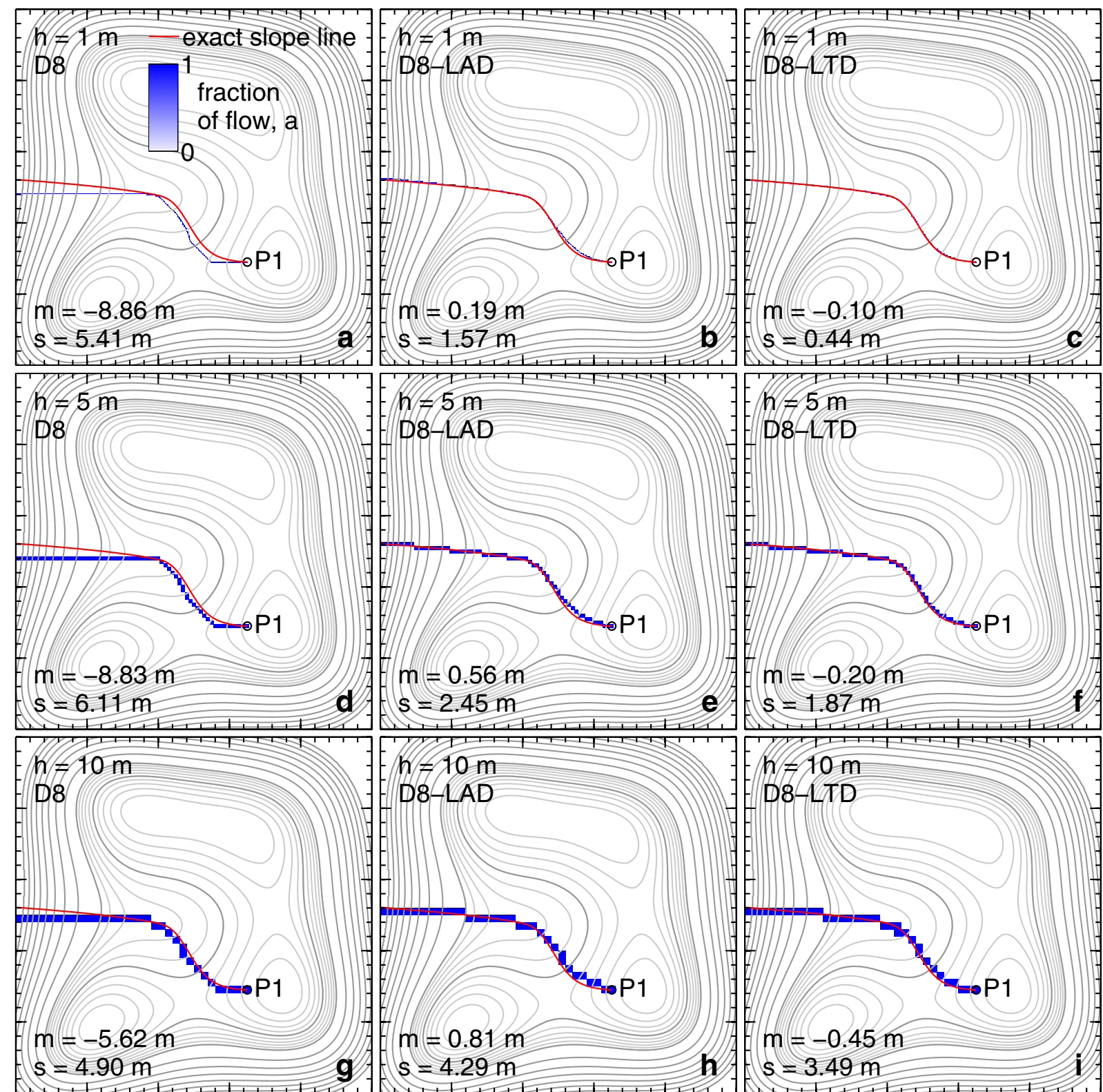

Figure 4. Propagation patterns predicted in grid digital elevation models having variable resolution ( $h=1 \mathrm{~m}$ in maps a, b, and $\mathrm{c} ; h=5 \mathrm{~m}$ in maps $\mathrm{d}$, e, and $\mathrm{f} ; h=10 \mathrm{~m}$ in maps $\mathrm{g}, \mathrm{h}$, and i) by using the D8 (maps a, d, and g), D8-LAD (maps b, e, and d), and D8-LTD (maps c, f, and i) methods. For any cell, the blue shade indicates the fraction of the cell source centered in P1 that propagates through that cell. The exact slope line beginning at point P1 is shown in red. Weighted means $m$ and standard deviations $s$ of the deviation distance between the centers of the cells of the propagation pattern and the exact slope line are reported in the bottom left corner of each map. The intervals between dark gray contour lines are equal to $50 \mathrm{~m}$, and each of these intervals is subdivided if necessary into five subintervals equal to $10 \mathrm{~m}$ by using four light gray contour lines.

$$
m=\frac{\sum_{i=1}^{N} d_{i} a_{i}}{\sum_{i=1}^{N} a_{i}},
$$

where the summations extend over the $N$ cells of the simulated propagation pattern beginning at a given point $(\mathrm{P} 1$, $\mathrm{P} 2$, or $\mathrm{P} 3), d_{i}(i=1, \cdots, N)$ is the distance with sign between the center of the $i$ th cell of the pattern and the exact slope line beginning at the given point (P1, P2, or

P3), and $a_{i}(i=1, \cdots, N)$ is the fraction of flow released at the cell source centered in the given point (P1, P2, or P3) that propagates through the $i$ th cell of the pattern. As sketched in Figure 6, the distance $d_{i}(i=1, \cdots, N)$ is computed as the minimum distance from the center of the $i$ th cell (e.g., C1, C2, C3, C4, C5, and C6 in Figure 6) and the exact slope line (red line in Figure 6). This minimum distance can occur along the normal to the exact slope line (e.g., distance of cells C1, C2, C3, and C4 in Figure 6) or 

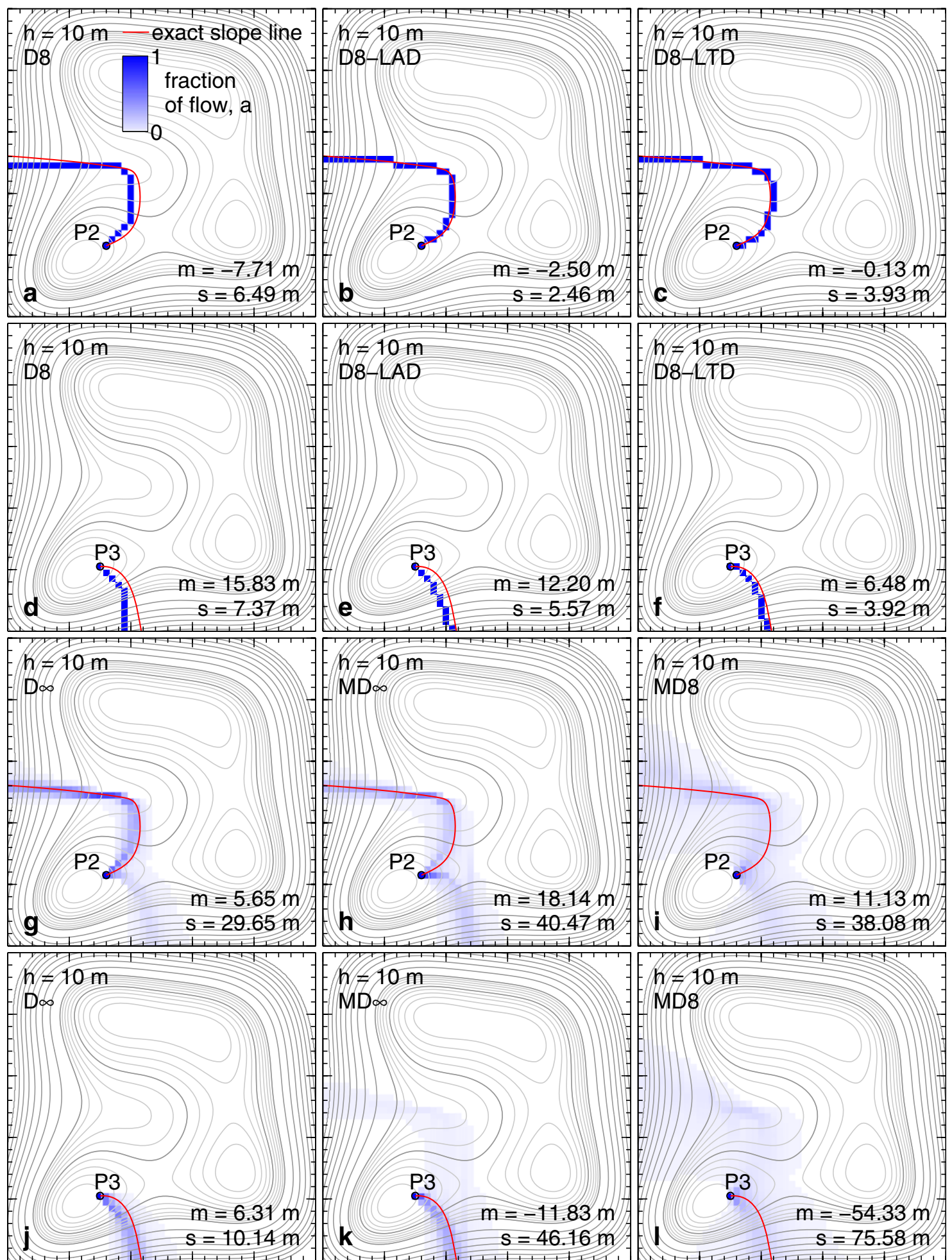

Figure 5. Propagation patterns predicted in grid digital elevation models having resolution $h=10 \mathrm{~m}$ by using the (maps a and d) D8, (maps b and h) D8-LAD, (maps c and f) D8-LTD, (maps g and j) D $\infty$, (maps $\mathrm{h}$ and $\mathrm{k}$ ) MD $\infty$, and (maps i and l) MD8 methods. For any cell, the blue shade indicates the fraction of the cell source centered in P2 (maps a, b, c, g, h, and i) or P3 (maps d, e, f, j, k, and l) that propagates through that cell. The exact slope lines beginning at points P2 and P3 are shown in red. Weighted means $m$ and standard deviations $s$ of the deviation distance between the centers of the cells of the propagation pattern and the exact slope line are reported in the bottom right corner of each map. The intervals between dark gray contour lines are equal to $50 \mathrm{~m}$, and each of these intervals is subdivided if necessary into five subintervals equal to $10 \mathrm{~m}$ by using four light gray contour lines. 


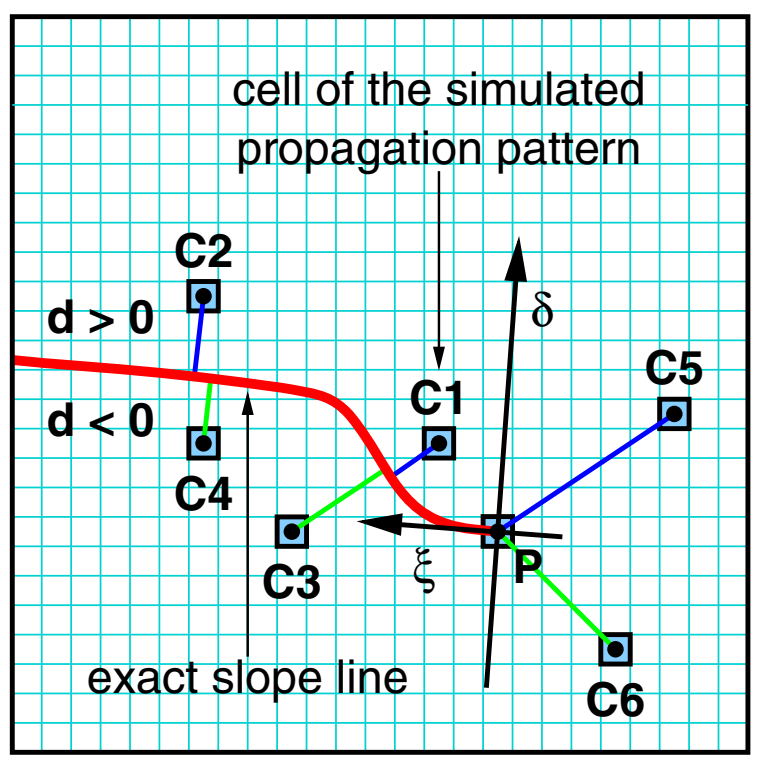

Figure 6. Sketch of an exact slope line initiating at point $\mathrm{P}$ (red line) and of the quantities involved for the computation of bias and dispersion (equations (44) and (45), respectively) with which this line is approximated by the cells of a simulated propagation pattern (e.g., light blue cells C1, C2, C3, C4, $\mathrm{C} 5$, and $\mathrm{C} 6$ ). For each of these cells, the deviation distance $d$ is computed as the minimum distance from the cell center and the exact slope line (blue and bright green lines for positive and negative values, respectively). This distance is computed along the normal to the exact slope line (e.g., distances of cells $\mathrm{C} 1, \mathrm{C} 2, \mathrm{C} 3$, and $\mathrm{C} 4$ ) or along the line connecting the cell center to the initial point $\mathrm{P}$ (e.g., distances of cells $\mathrm{C} 5$ and C6).

along the line connecting the cell center to the given initial point P (e.g., cells C5 and C6 in Figure 6). The sign of $d_{i}(i=1$, $\cdots, N)$ is determined by considering the local coordinate system $\xi \delta$ defined in such a way that the origin is lying on the exact slope line, the $\xi$-axis is tangent to the exact slope line, and the $\delta$-axis is normal to the exact slope line. The value of $d_{i}(i=1, \cdots, N)$ is considered to be positive when the cell center lies to the right-hand side of an observer traveling downslope along the exact slope line $(\delta>0$, blue lines in Figure $6)$, null when it lies on the exact slope line $(\delta=0)$, negative when it lies to the left-hand side of the exact slope line $(\delta<0$, bright green lines in Figure 6$)$. When the distance $d_{i}(i=1, \cdots, N)$ is computed along the line connecting the cell center and the given initial point $\mathrm{P}$, the sign is determined by considering the coordinate system $\xi \delta$ having origin in $\mathrm{P}$. The weighted mean $m$ given by equation (44) provides a measure of the bias with which the simulated propagation pattern reproduces or develops around the exact slope line.

[49] The second metric is the weighted standard deviation of the deviation distance between the cells of the simulated propagation pattern and the exact slope line. This standard deviation is defined as

$$
s=\sqrt{\frac{N \sum_{i=1}^{N} a_{i}\left(d_{i}-m\right)^{2}}{(N-1) \sum_{i=1}^{N} a_{i}}} .
$$

[50] The weighted standard deviation $s$ given by equation (45) provides a measure of dispersion of the distances $d_{i}(i=1, \cdots, N)$ around their mean value $m$. The mean distance $m$ (equation (44)) and the standard deviation $s$ (equation (45)) are suitable metrics for the evaluation of the simulated propagation patterns' capabilities to reproduce or develop around exact slope lines. A perfect reproduction of the exact slope line provides $m=0$ and $s=0$, implying that all the centers of the grid cells of the propagation pattern lay on the exact slope line. Propagation patterns providing small values of $m$ and significantly high values of $s$ are unbiased, but may only be suitable to describe dispersive flows. Propagation patterns providing significantly high values of $m$ are biased, and may therefore be unsuitable to describe both nondispersive and dispersive flows. The values of $m$ and $s$ obtained for the numerical cases considered in section 4 are reported in the maps of Figures 3-5. The variations of $m$ and $s$ with grid cell size $h$ $(h=0.01,0.05,0.1,0.5,1,5,10,20,30,40,50 \mathrm{~m})$, for the slope lines beginning at points $\mathrm{P} 1, \mathrm{P} 2$, and $\mathrm{P} 3$, and for the various slope direction methods considered in the present study (D8, D8-LAD, D8-LTD, D $\infty, \mathrm{MD} \infty$, and MD8 methods) are reported in Figure 7 . The mean absolute value of the mean distance $m$, namely $\langle|m|\rangle$, and the mean value of the standard deviation $s$, namely $\langle s\rangle$, computed for each grid cell size $h$ over the selected test cases (i.e., the slope lines beginning at points $\mathrm{P} 1, \mathrm{P} 2$, and $\mathrm{P} 3$ ) are reported in Table 2 and Figure 8 . The domain used in the maps reported in Figures 4 and $5(0 \leq x \leq 500 \mathrm{~m}$ and $0 \leq y \leq 500 \mathrm{~m})$ are also used for the computation of the values of $m$ and $s$ reported in Figure 7, Table 2, and Figure 8.

\section{Discussion}

[51] The D8-LTD method provides slope lines in grid digital elevation models that approximate consistently the exact slope lines as the grid cell size goes to zero. This is shown analytically for any $C^{2}$ surface by using mathematical analysis methods (section 3, Figure 1). The use of cumulative transverse deviations is found to be the key factor allowing for globally unbiased approximations of slope lines (section 3, Remark 3.7). The D8 method does not display the same capabilities of the D8-LTD method because it performs for each grid cell a local analysis rather than a path-based analysis (sections 2 and 3, Remark 3.7). The D8-LAD method is not as accurate as the D8-LTD method because angular deviations are unsuitable for path-based analyses along slope lines as compared to transverse deviations (section 3, Remark 3.8). To demonstrate these analytical results, numerical experiments are performed by considering a synthetic drainage system based on the Himmelblau function (Figure 2). The D8-LTD method provides close approximations of the exact slope lines when a small grid cell size is used. In the case illustrated in Figure 3, $h=0.50 \mathrm{~m}, m=-0.07 \mathrm{~m}$, and $s=0.23 \mathrm{~m}$. The D8 method may provide a close approximation of the exact slope line from point P1 where the terrain is markedly convergent (e.g., along the valley located around the central part of the slope line reported in Figure 3), but it is unable to provide predictions that approximate closely this exact slope line over a divergent or feebly convergent terrains (e.g., around the upper and lower parts of the slope line reported in Figure 3). The overall performance of the D8 method is represented in this case by the values $m=-8.69 \mathrm{~m}$ and $s=5.24$ 

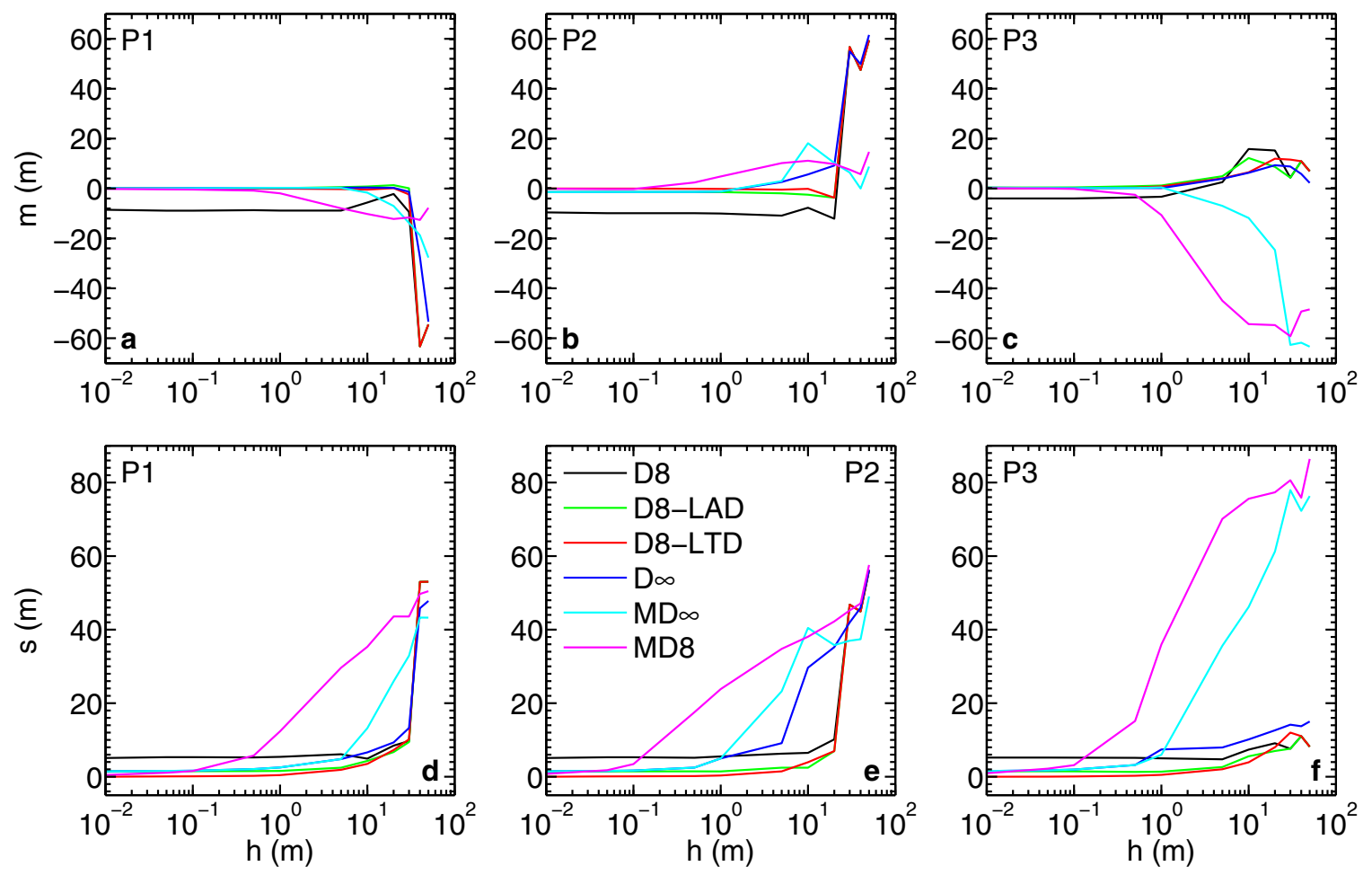

Figure 7. Weighted means $m$ and standard deviations $s$ of the deviation distance $d$ between the centers of the cells of the predicted propagation patterns and the exact slope lines beginning at point (a and d) P1, (b and e) P2, and (c and f) P3 (equations (44) and (45)). The D8, D8-LAD, D8-LTD, D $\infty, \mathrm{MD} \infty$, and MD8 methods and the grid cell sizes $h=0.01,0.05,0.1,0.5,1,5,10,20,30,40$, and $50 \mathrm{~m}$ are considered.

m. The D8-LAD method outperforms significantly the D8 method $(m=0.10 \mathrm{~m}$ and $s=1.53 \mathrm{~m})$, but it does not ensure the same degree of accuracy of the D8-LTD method (Figure 3).

[52] It is found numerically that the D8-LTD method provides better approximations of exact slope lines than the D8 and D8-LAD methods over a wide range of grid cell sizes (Figures 4, 5, 7, and 8; Table 2). The mean distance $m$ displayed by the D8 method ranges from -8.86 to 15.83 $\mathrm{m}$ as $h$ goes from 1 to $10 \mathrm{~m}$ (Figures $4 \mathrm{a}, 4 \mathrm{~d}, 4 \mathrm{~g}, 5 \mathrm{a}$, and $5 \mathrm{~d}$ ), revealing that the limitations of the D8 method are relevant even when small grid cell sizes are used to provide a detailed description of land surface topography. The values of the mean distance $m$ displayed by the D8-LTD method ranges from -0.10 to $6.48 \mathrm{~m}$ as $h$ goes from 1 to $10 \mathrm{~m}$ (Figures 4c, 4f, 4i, 5c, and 5f), revealing that the use of the D8-LTD method is especially justified when an accurate description of land surface topography is available. This does not necessarily mean that a high-resolution digital elevation model must be available, but rather that the

Table 2. Mean Absolute Value of the Mean Deviation Distance, $\langle|m|\rangle$, and Mean Standard Deviation, $\langle s\rangle$, Computed Over the Selected Test Cases (Slope Lines Beginning at Points P1, P2, and P3)

\begin{tabular}{|c|c|c|c|c|c|c|c|c|c|c|c|}
\hline \multicolumn{12}{|c|}{$h(\mathrm{~m})$} \\
\hline Method & 0.01 & 0.05 & 0.1 & 0.5 & 1 & 5 & 10 & 20 & 30 & 40 & 50 \\
\hline \multicolumn{12}{|c|}{ Mean Absolute Value of the Mean Deviation Distance $\langle|m|\rangle(\mathrm{m})$} \\
\hline D8 & 7.35 & 7.58 & 7.58 & 7.37 & 7.40 & 7.41 & 9.72 & 9.84 & 23.49 & 40.57 & 40.28 \\
\hline D8-LAD & 0.64 & 0.64 & 0.65 & 0.82 & 0.94 & 2.47 & 5.17 & 4.57 & 20.38 & 40.57 & 40.28 \\
\hline D8-LTD & 0.00 & 0.03 & 0.05 & 0.17 & 0.37 & 1.58 & 2.36 & 5.26 & 23.56 & 40.57 & 40.28 \\
\hline $\mathrm{D} \infty$ & 0.62 & 0.58 & 0.56 & 0.59 & 0.55 & 2.27 & 4.12 & 6.27 & 21.68 & 27.74 & 39.06 \\
\hline $\mathrm{MD} \infty$ & 0.62 & 0.58 & 0.56 & 0.60 & 0.64 & 3.39 & 10.54 & 13.98 & 27.61 & 26.83 & 33.28 \\
\hline MD8 & 0.14 & 0.20 & 0.26 & 1.93 & 5.81 & 21.03 & 25.23 & 25.58 & 26.12 & 22.54 & 23.62 \\
\hline \multicolumn{12}{|c|}{ Mean Standard Deviation $\langle s\rangle(\mathrm{m})$} \\
\hline D8 & 5.12 & 5.27 & 5.27 & 5.18 & 5.31 & 5.71 & 6.26 & 9.24 & 21.37 & 36.31 & 39.13 \\
\hline D8-LAD & 1.48 & 1.48 & 1.48 & 1.43 & 1.46 & 2.52 & 4.11 & 6.89 & 21.28 & 36.31 & 39.13 \\
\hline D8-LTD & 0.00 & 0.07 & 0.09 & 0.23 & 0.43 & 1.79 & 3.78 & 7.42 & 22.99 & 36.31 & 39.13 \\
\hline $\mathrm{D} \infty$ & 1.51 & 1.63 & 1.76 & 2.58 & 4.97 & 7.29 & 15.47 & 19.07 & 23.13 & 35.16 & 39.68 \\
\hline $\mathrm{MD} \infty$ & 1.51 & 1.63 & 1.76 & 2.58 & 4.49 & 21.22 & 33.27 & 41.00 & 49.28 & 50.98 & 56.21 \\
\hline MD8 & 0.73 & 1.68 & 2.71 & 12.81 & 24.05 & 44.84 & 49.66 & 54.39 & 56.47 & 57.56 & 64.82 \\
\hline
\end{tabular}



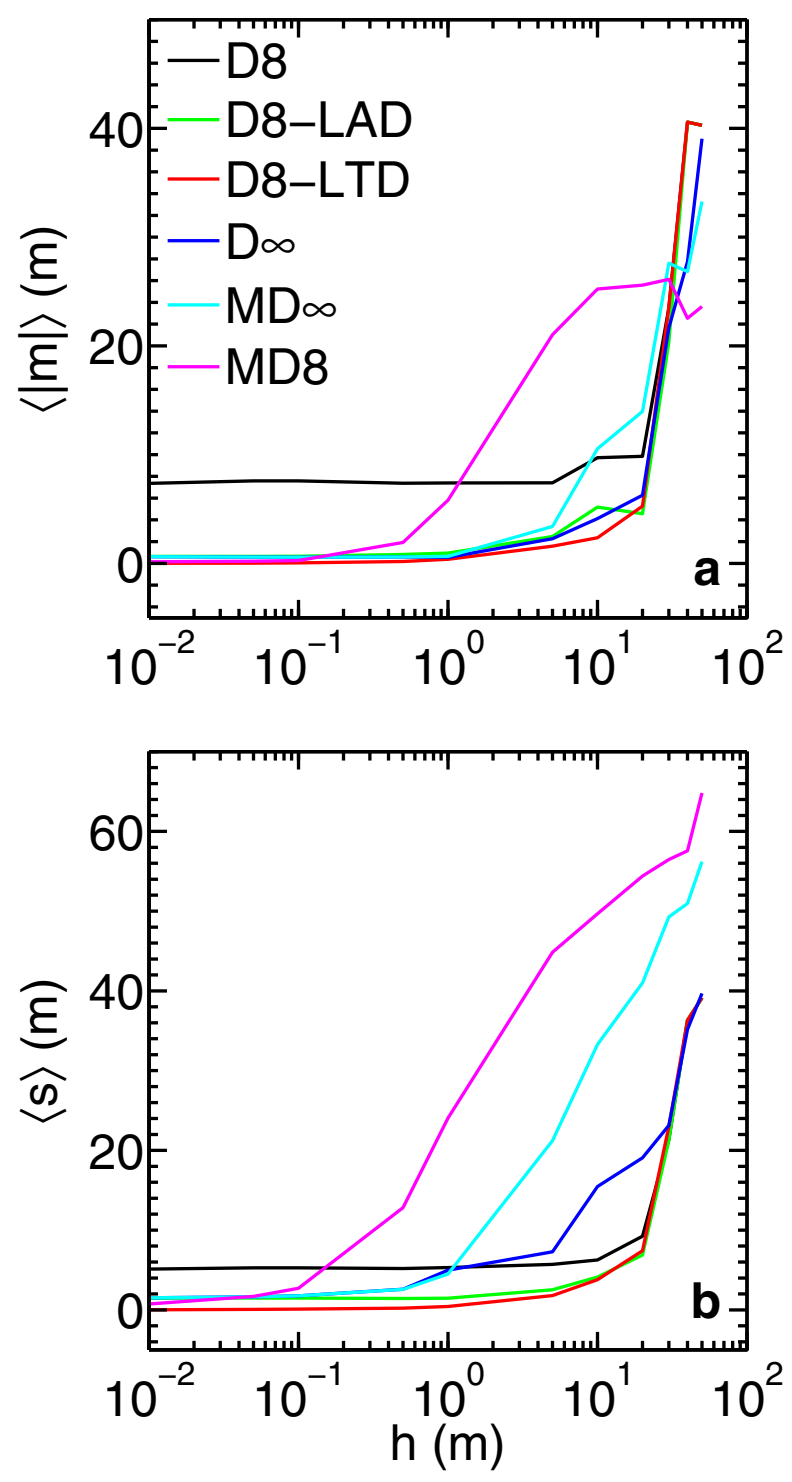

Figure 8. (a) Mean absolute value of the mean deviation distance $\langle|m|\rangle$ and (b) mean standard deviation $\langle s\rangle$ over the selected test cases (slope lines beginning at points P1, P2, and P3) for the D8, D8-LAD, D8-LTD, D $\infty, \mathrm{MD} \infty$, and MD8 methods and the grid cell sizes $h=0.01,0.05,0.1,0.5$, $1,5,10,20,30,40$, and $50 \mathrm{~m}$ (Table 2).

resolution of the digital elevation model should be adequate to describe the spatial variability of the considered terrain topography. This is clearly not the case of the resolutions $h \geq 40 \mathrm{~m}$ used to describe the sloping surface considered in section 4 , where equally unsatisfactory values of $m$ and $s$ are obtained from the D8, D8-LAD, and D8-LTD methods (Table 2). Further research is needed to identify the maximum grid cell size required to describe adequately a given land surface topography and related slope lines. An intermediate behavior is displayed by the D8-LAD method, which displays values of $m$ relatively more uniform over the investigated range of $h$. As shown in Figures 4b, 4e, 4h, $5 \mathrm{~b}$, and $5 \mathrm{e}, m$ ranges from 0.19 to $12.20 \mathrm{~m}$ as $h$ goes from 1 to $10 \mathrm{~m}$. As also reported in Figure 7, Table 2, and Figure 8 , the D8-LTD method outperforms significantly the D8 method for small grid cell sizes $(h \leq 20 \mathrm{~m}$ in this application), and it is normally not outperformed by other nondispersive methods for any grid cell size. One can note that for $h \geq 40 \mathrm{~m}$, the MD $\infty$ and MD8 (fully dispersive) methods provides smaller values of $\langle|m|\rangle$ than other methods $(\langle|m|\rangle<33.28 \mathrm{~m}$ in Table 2 and Figure $8 \mathrm{a})$. It is noted, however, that these results are obtained by allowing a large dispersion to occur $(\langle s\rangle \geq 50.98 \mathrm{~m}$ in Table 2 and Figure $8 b)$.

[53] As reported in Figure 5 for $h=10 \mathrm{~m}$, the D8-LTD method ( $m$ ranging from -0.13 to $6.48 \mathrm{~m}$ and $s$ ranging from 3.92 to $3.93 \mathrm{~m}$ in Figures $5 \mathrm{c}$ and $5 \mathrm{f}$ ) provides the most accurate approximations of the exact slope lines as compared to the other methods ( $m$ ranging from -54.33 to $18.14 \mathrm{~m}$ and $s$ ranging from 2.46 to $75.58 \mathrm{~m}$ in Figures 5a, $5 \mathrm{~b}, 5 \mathrm{~d}, 5 \mathrm{e}$, and $5 \mathrm{~g}-5 \mathrm{l})$. Specifically, the $\mathrm{D} \infty, \mathrm{MD} \infty$, and MD8 (dispersive) methods display larger values of $s$ (ranging from 10.14 to $75.5 \mathrm{~m}$ in Figures $5 \mathrm{~g}-5 \mathrm{l}$ ) and therefore these methods can only be used to describe overland flows dominated by dispersion. Among these dispersive methods, the $\mathrm{D} \infty$ method yields more unbiased simulated propagation patterns ( $m$ ranging from 5.65 to $6.31 \mathrm{~m}$ in Figures $5 \mathrm{~g}$ and $5 \mathrm{j}$ ) than the $\mathrm{MD} \infty$ and MD8 methods ( $m$ ranging from -54.33 to $18.14 \mathrm{~m}$ in Figures $5 \mathrm{~h}, 5 \mathrm{i}, 5 \mathrm{k}$, and 51 ). As also reported in Orlandini et al. [2012], the description of dispersive overland flows developing around slope lines remains an open issue that needs to be addressed in future investigations.

[54] The results reported in Figure 7, Table 2, and Figure 8 demonstrate that the D8-LTD method displays values of $m$ and $s$ that tend to zero as the grid cell size $h$ goes to zero $(\langle|m|\rangle=0.00 \mathrm{~m}$ and $\langle s\rangle=0.00 \mathrm{~m}$ for $h=0.01 \mathrm{~m}$ in Table 2 and Figure 8), confirming that the D8-LTD method predicts consistently the exact slope lines. Small values of $\langle|m|\rangle$ and $\langle s\rangle$, namely $\langle|m|\rangle \leq 5.26 \mathrm{~m}$ and $\langle s\rangle \leq 7.42 \mathrm{~m}$, are displayed by the D8-LTD method for $h \leq 20 \mathrm{~m}$ (Table 2 and Figure $8)$. For $h>20 \mathrm{~m}$, the values of $\langle|m|\rangle$ and $\langle s\rangle$ displayed by the D8-LTD method may become large $(\langle|m|\rangle \geq 23.56 \mathrm{~m}$ and $\langle s\rangle \geq 22.99 \mathrm{~m}$ in Table 2 and Figure 8 ), revealing that the use of excessively large grid cell sizes $h$ may undermine the reliability of the slope lines obtained (Figures $7 \mathrm{a}, 7 \mathrm{~b}$, $7 \mathrm{~d}$, and 7e). The D8-LAD method is significantly outperformed by the D8-LTD method as the grid cell size $h$ goes to zero (Table 2 and Figure 8 ). For values of the grid cell size $h$ that go to zero, the D8 method provides larger values of $m$ (Figures $7 \mathrm{a}-7 \mathrm{c},\langle|m|\rangle=7.35 \mathrm{~m}$ for $h=1 \mathrm{~m}$ in Table 2 and Figure $8 \mathrm{a}$ ) than other methods, revealing that the propagation patterns simulated by the D8 method are significantly biased even for small grid cell sizes. The values of $m$ provided by the D8 method become comparable with those provided by other methods for large grid cell sizes (Figures $7 \mathrm{a}-7 \mathrm{c},\langle|m|\rangle \geq 23.49 \mathrm{~m}$ for $h \geq 30 \mathrm{~m}$ in Table 2 and Figure $8 \mathrm{a})$. In these cases, however, all the approximations of the exact slope lines provided by the considered slope direction methods are poor as a result of an inadequate description of the surface topography. The D8 method provides larger values of $s$ than other nondispersive methods for small values of the grid cell size $(\langle s\rangle \geq 5.12 \mathrm{~m}$ for $h \leq 1 \mathrm{~m}$ in Table 2 and Figure $8 \mathrm{~b}$ ) and comparable with those provided by other nondispersive methods for large values of the grid cell size $(\langle s\rangle \geq 21.37 \mathrm{~m}$ for $h \geq 30 \mathrm{~m}$ in Table 2 and Figure $8 b)$. 
[55] In the range $1 \leq h \leq 20 \mathrm{~m}$, the values of $m$ displayed by the $\mathrm{D} \infty$ method $(0.55 \leq\langle|m|\rangle \leq 6.27 \mathrm{~m}$ in Table 2) are comparable with those provided by the D8-LTD method $(0.37 \leq\langle|m|\rangle \leq 5.26 \mathrm{~m}$ in Table 2$)$, revealing that the propagation patterns simulated by the $\mathrm{D} \infty$ method may be affected by little bias (Figures $7 a-7 c$ and $8 a$ ). As expected, however, the dispersion provided by the $\mathrm{D} \infty$ method $(4.97 \leq\langle s\rangle \leq 19.07 \mathrm{~m}$ in Table 2$)$ is larger than the one provided by D8-LTD (nondispersive) method $(0.43 \leq\langle s\rangle \leq 7.42 \mathrm{~m}$ in Table 2 , Figures $7 \mathrm{~d}-7 \mathrm{f}$ and $8 \mathrm{~b})$. The $\mathrm{MD} \infty$ (fully dispersive) method provides simulated propagation patterns affected by significant bias $(\langle|m|\rangle \geq 10.54 \mathrm{~m}$ for $h \geq 10 \mathrm{~m}$ in Table 2 and Figure $8 \mathrm{a}$ ) and large dispersion $(\langle s\rangle \geq 21.22 \mathrm{~m}$ for $h \geq 5 \mathrm{~m}$ in Table 2 and Figure $8 \mathrm{~b}$ ). It is, however, noted that results comparable with those provided by the $\mathrm{D} \infty$ method are provided by the $\mathrm{MD} \infty$ method as the grid cell size $h$ goes to zero $(\langle|m|\rangle \leq 0.64 \mathrm{~m}$ and $\langle s\rangle \leq 4.49 \mathrm{~m}$ for $h \leq 1 \mathrm{~m}$ in Table 2 and Figure 8 ). The MD8 (fully dispersive) method provides simulated propagation patterns affected by significant bias $(\langle|m|\rangle \geq 21.03 \mathrm{~m}$ for $h \geq 5 \mathrm{~m}$ in Table 2 and Figure $8 \mathrm{a})$ and large dispersion $(\langle s\rangle \geq 24.05 \mathrm{~m}$ for $h \geq 1 \mathrm{~m}$ in Table 2 and Figure $8 \mathrm{~b})$. As the grid cell size $h$ goes to zero, the MD8 method displays less bias $(\langle|m|\rangle=0.14 \mathrm{~m}$ for $h=0.01 \mathrm{~m}$ in Table 2 and Figure $8 \mathrm{a})$ and dispersion $(\langle s\rangle=0.73 \mathrm{~m}$ for $h=$ $0.01 \mathrm{~m}$ in Table 2 and Figure $8 \mathrm{~b}$ ) than all the other methods with except for the D8-LTD method.

\section{Conclusions}

[56] The analysis carried out in this study reveals that: (1) The D8-LTD (nondispersive) method provides slope lines in grid digital elevation models that consistently approximate the exact slope lines as the grid cell size goes to zero (sections 3 and 4; Figures 3, 4, 7, and 8; Table 2). (2) At least for grid cell sizes that are not excessively large (up to $20 \mathrm{~m}$ in this application), the D8-LTD method outperforms significantly the D8 and D8-LAD (nondispersive) methods (sections 3 and 4; Figures 3, 4, 5, 7, and 8; Table 2). (3) The $\mathrm{D} \infty$ (moderately dispersive) method and the $\mathrm{MD} \infty$ (fully dispersive) method provide propagation patterns that develop around the exact slope lines in a reasonably unbiased manner, whereas the MD8 (fully dispersive) method may produce unreliable propagation patterns (section 4; Figures 5, 7, and 8; Table 2). From these results, it can be argued that the D8-LTD method should be used in preference to the standard D8 method in order to obtain slope lines that are only limited in reliability by the detail of topographic data, and not by the accuracy of the slope direction method applied. While the $\mathrm{D} \infty$ and $\mathrm{MD} \infty$ methods can be used to describe overland flow dispersion, the D8-LAD and MD8 methods do not seem to offer significant advantages over the other methods. Future research is needed to identify criteria for the determination of the range of grid cell sizes that ensure meaningful representations of land surface topography, slope lines, and propagation patterns in grid digital elevation models. These meaningful representations of land surface topography and related attributes are the essential basis that needs to be considered to develop hydrodynamically realistic descriptions of overland flows along complex hillslope systems.

\section{Appendix A : Proof of Lemma 3.2}

[57] Since $\hat{\mathbf{x}}$ is bounded, we can find two constants $m$ and $M$ in such a way that equation (21) holds for $\mathbf{x}=\hat{\mathbf{x}}(t)$. Now, from (23), we infer the existence of a scalar, measurable function $\lambda(t)$ such that $\hat{\mathbf{x}}^{\prime}(t)=\lambda(t) \nabla z(\hat{\mathbf{x}}(t))$. In particular, we get $|\lambda(t)| \leq L / m$, where $L>0$ is a Lipschitz constant for $\hat{\mathbf{x}}$, that is: $\left\|\hat{\mathbf{x}}^{\prime}(t)\right\| \leq L$ for almost every $t \in[0, r]$. On the other hand, equation (24) entails $\lambda(t) \leq-c / m^{2}$, so that $-L / m \leq \lambda(t) \leq-c / m^{2}$. Hence, the function $\Lambda(t)=-\int_{0}^{t} \lambda(\tau) d \tau$ is one to one on $[0, r]$, and admits a differentiable inverse function $s$, whose derivative fulfills the identity $\lambda(s(\tau)) s^{\prime}(\tau)=-1$ for any $\tau \in J=[0, \Lambda(r)]$. Now it is possible to see that the function $\mathbf{w}(\tau)=\hat{\mathbf{x}}(s(\tau))$ solves the differential equation $\mathbf{w}^{\prime}=-\nabla z(\mathbf{w})$ on $J$, together with the initial condition $\mathbf{w}(0)=\mathbf{p}_{0}$. Hence, $\mathbf{w}$ $=\mathbf{x}$ on $J$, where $\mathbf{x}$ comes from equations (8) and (9) with $\mathbf{x}_{0}=\mathbf{p}_{0}$. In particular: $\hat{\mathbf{x}}([0, r])=\mathbf{w}(J)=\mathbf{x}(J) \subseteq \Gamma$.

\section{Appendix B : Proof of Lemma 3.4}

[58] Let $\mathbf{w}_{h}^{i}(\mathbf{p})$ denote the unit vector of $\mathbf{v}_{h}^{i}(\mathbf{p})$, and put $\mathbf{v}_{h}=\mathbf{v}_{h}^{i(h)}(\mathbf{p}), \mathbf{w}_{h}=\mathbf{w}_{h}^{i(h)}(\mathbf{p})$, where $i(h)$ comes from equation (11). Let $j(h)$ be such that the vector $\mathbf{u}_{h}=\mathbf{v}_{h}^{j(h)}(\mathbf{p})$ fulfills the equality $\Delta_{h}\left(\mathbf{p} ;-\mathbf{w}_{h}\right)=\mathbf{u}_{h} \cdot\left(-\mathbf{w}_{h}\right)$. Then

$$
\left\|\mathbf{v}_{h}-\mathbf{u}_{h}\right\| \leq 8 K h, \quad\left\|\mathbf{v}_{h}\right\| \geq m / 2, \quad 0<h \leq h^{*} .
$$

[59] Indeed, from equation (35) we argue that the distance of both vectors $\mathbf{v}_{h}$ and $\mathbf{u}_{h}$ from $\nabla z(\mathbf{p})$ does not exceed $4 \mathrm{Kh}$, so as to infer the first inequality of equation (B1). On the other hand, the latter follows from equation (35) as well thanks to our choice of $h^{*}$ and the first inequality in equation (21). Furthermore

$$
\begin{gathered}
\left\|\mathbf{T}_{h}(\mathbf{p})+\mathbf{w}_{h}\right\|^{2}-2=2 \mathbf{w}_{h} \cdot \mathbf{T}_{h}(\mathbf{p})= \\
=2\left\|\mathbf{v}_{h}\right\|^{-1} \Delta_{h}\left(\mathbf{p} ; \mathbf{T}_{h}(\mathbf{p})\right) \leq \\
\leq 2\left\|\mathbf{v}_{h}\right\|^{-1} \Delta_{h}\left(\mathbf{p} ;-\mathbf{w}_{h}\right)=-2\left\|\mathbf{v}_{h}\right\|^{-2}\left(\mathbf{u}_{h} \cdot \mathbf{v}_{h}\right)= \\
=2\left\|\mathbf{v}_{h}\right\|^{-2}\left(\mathbf{v}_{h}-\mathbf{u}_{h}\right) \cdot \mathbf{v}_{h}-2 .
\end{gathered}
$$

[60] The first equality in equation (B2) holds because both $\mathbf{T}_{h}(\mathbf{p})$ and $\mathbf{w}_{h}$ are unit vectors, while the second one and the inequality are due to equation (11). Hence

$$
\left\|\mathbf{T}_{h}(\mathbf{p})+\mathbf{w}_{h}\right\| \leq \sqrt{2}\left\|\mathbf{v}_{h}\right\|^{-1 / 2} \sqrt{\left\|\mathbf{v}_{h}-\mathbf{u}_{h}\right\|} \leq 4 \sqrt{2 K h / m}
$$

where the last inequality follows from equation (B1). Now, let us put $i=i(h)$ in equation (35), and recall that $\mathbf{w}_{h}$ and $\mathbf{T}(\mathbf{p})$ are the unit vectors of $\mathbf{v}_{h}$ and $\nabla z(\mathbf{p})$, respectively. Thanks to the first inequality in equation (21), we argue that the norm of $\mathbf{w}_{h}+\mathbf{T}(\mathbf{p})$ is bounded from above by the number $8 \mathrm{Kh} / \mathrm{m}$. Even more so, it cannot exceed its square root $2 \sqrt{2 K h / m}$ since the inequality $h \leq h^{*}$ entails $8 K h / m \leq 1$. Then, by virtue of equation (B3), the last estimate and the definition of $C$ we get our claim as follows:

$$
\begin{gathered}
\left\|\mathbf{T}_{h}(\mathbf{p})-\mathbf{T}(\mathbf{p})\right\| \leq\left\|\mathbf{T}_{h}(\mathbf{p})+\mathbf{w}_{h}\right\|+\left\|\mathbf{w}_{h}+\mathbf{T}(\mathbf{p})\right\| \leq \\
\leq 4 \sqrt{2 K h / m}+2 \sqrt{2 K h / m}=C \sqrt{h} .
\end{gathered}
$$




\section{Appendix C: Proof of Lemma 3.5}

[61] For any $t \in[0, r]$, there exists just one integer $k$ such that $t \in J(n, k)$. Then, we put $\tau_{n}(t)=(k-1) r / n$. According to this notation, one can write

$$
\begin{gathered}
\left|\tau_{n}(t)-t\right| \leq r / n, \\
\mathbf{x}_{n}\left(\tau_{n}(t)\right)=\mathbf{x}_{n}(k r / n)=\mathbf{p}_{k}(r / n), \\
\hat{\mathbf{q}}_{n}(t)=\mathbf{T}_{r / n}\left(\mathbf{p}_{k}(r / n)\right)=\mathbf{T}_{r / n}\left(\mathbf{x}_{n}\left(\tau_{n}(t)\right) ; r / n\right), \\
\left\|\hat{\mathbf{q}}_{n}(t)-\mathbf{T}\left(\mathbf{x}_{n}\left(\tau_{n}(t)\right)\right)\right\| \leq C \sqrt{r / n} .
\end{gathered}
$$

[62] In particular, equation (C4) follows from equation (C3) and Lemma 3.4, provided that in the latter we put $h=r / n$. Furthermore, as $n \rightarrow+\infty$ :

$$
\begin{gathered}
\sup \left\{\left\|\mathbf{x}_{n}\left(\tau_{n}(t)\right)-\hat{\mathbf{x}}(t)\right\| ; 0 \leq t \leq r\right\} \rightarrow 0, \\
\sup \left\{\left\|\mathbf{T}\left(\mathbf{x}_{n}\left(\tau_{n}(t)\right)\right)-\hat{\mathbf{q}}(t)\right\| ; 0 \leq t \leq r\right\} \rightarrow 0 .
\end{gathered}
$$

[63] Indeed, equations (30) and (C1) entail (C5) because the convergence in equation (30) is uniform. On the other hand, (C5) implies in turn (C6), thanks to the definition of $\hat{\mathbf{q}}$ and the continuity of $\mathbf{T}$. Finally, our claim follows from equations (C4) and (C6).

\section{Appendix D: Proof of Lemma 3.6}

[64] From the first estimate in equation (29) and Lemma 3.5 we argue that, as $n \rightarrow+\infty$,

$$
\int_{0}^{t} \mathbf{x}_{n}^{\prime}(\tau) \cdot\left(\mathbf{A} \hat{\mathbf{q}}_{n}(\tau)-\mathbf{A} \hat{\mathbf{q}}(\tau)\right) d \tau \rightarrow 0
$$

[65] On the other hand, by putting $s=0$ and $\mathbf{v}=\mathbf{A} \hat{\mathbf{q}}$ in (31) we get

$$
\int_{0}^{t} \mathbf{x}_{n}^{\prime}(\tau) \cdot \mathbf{A} \hat{\mathbf{q}}(\tau) d \tau \rightarrow \int_{0}^{t} \hat{\mathbf{x}}^{\prime}(\tau) \cdot \mathbf{A} \hat{\mathbf{q}}(\tau) d \tau
$$

[66] Finally, when combining (34), (D1), and (D2), we get $\int_{0}^{t} \hat{\mathbf{x}}^{\prime}(\tau) \cdot \mathbf{A} \hat{\mathbf{q}}(\tau) d \tau=0$ for any $t \in[0, r]$. Then, actually, $\hat{\mathbf{x}}^{\prime}$ $\cdot \mathbf{A} \hat{\mathbf{q}}=0$ on $[0, r]$, so that, according to our definition of $\mathbf{A}$, $\hat{\mathbf{x}}^{\prime}(t) \| \hat{\mathbf{q}}(t)$. Hence (23) holds true, since $\hat{\mathbf{q}}(t) \| \nabla z(\hat{\mathbf{x}}(t))$. Furthermore, thanks to the first inequality in (17), the scalar product $\mathbf{p}_{n}(t)=\mathbf{x}_{n}^{\prime}(t) \cdot \hat{\mathbf{q}}_{n}(t)$ is bounded from below by $1 / \sqrt{2}$ : then $\int_{s}^{t} \mathbf{p}_{n}(\tau) d \tau \geq(t-s) / \sqrt{2}$ whenever $0 \leq s<\tau \leq r$. Now, by virtue of Lemma 3.5 and (31), the left-hand side of the previous evaluation is easily shown to converge to $\int_{s}^{t} \mathbf{p}(\tau) d \tau$, where $\mathbf{p}(t)=\hat{\mathbf{x}}^{\prime}(t) \cdot \hat{\mathbf{q}}(t)$, so the limit inequality shows that the integral of $\mathbf{p}(\tau)-(1 / \sqrt{2})$ over $[s, t]$ is nonnegative: since $s$ and $t$ are arbitrary, we necessarily infer $\mathbf{p}(\tau) \geq 1 / \sqrt{2}$ for any $\tau \in[0, r]$. Now equation (24) is surely satisfied as well, provided we take $c=m / \sqrt{2}$, where $m$ comes from equation (21).

[67] Acknowledgments. This study was carried out under the research program PRIN 2010-2011 (grant 2010JHF437) funded by the Italian Ministry of Education, University, and Research. The authors are grate- ful to the Associate Editor and the three anonymous reviewers for comments that led to improvements in the manuscript.

\section{References}

Brezis, H. (2010), Functional Analysis, Sobolev Spaces and Partial Differential Equations, 599 pp., Springer, New York

Cayley, A. (1859), On contour and slope lines, London Edinburgh Dublin Philos. Mag. J. Sci., Ser. 4, 18(120), 264-268.

Costa-Cabral, M., and S. J. Burges (1994), Digital elevation model networks (DEMON): A model of flow over hillslopes for computation of contributing and dispersal areas, Water Resour. Res., 30(6), 1681-1692.

Fairfield, J., and P. Leymarie (1991), Drainage networks from grid digital elevation models, Water Resour. Res., 27(5), 709-717.

Freeman, T. G. (1991), Calculating catchment area with divergent flow based on a regular grid, Comput. Geosci., 17(3), 413-422.

Gallant, J. C., and M. F. Hutchinson (2011), A differential equation for specific catchment area, Water Resour. Res., 47, W05535, doi:10.1029/ 2009WR008540.

Grimaldi, S., F. Nardi, F. Di Benedetto, E. Istanbulluoglu, and R. L. Bras (2007), A physically-based method for removing pits in digital elevation models, Adv. Water Resour., 30(10), 2151-2158.

Himmelblau, D. M. (1972), Applied Nonlinear Programming, McGrawHill, New York.

Lea, N. L. (1992), An aspect driven kinematic routing algorithm, in Overland Flow: Hydraulics and Erosion Mechanics, edited by A. J. Parsons and A. D. Abrahams, pp. 393-407, Chapman and Hall, New York.

Martz, L. W., and J. Garbrecht (1992), Numerical definition of drainage network and subcatchment areas from digital elevation models, Comput. Geosci., 18(6), 747-761

Maxwell, J. C. (1870), On hills and dales, London Edinburgh Dublin Philos. Mag. J. Sci., Ser. 4, 40(269), 421-425.

Moore, I. D., and R. B. Grayson (1991), Terrain-based catchment partitioning and runoff prediction using vector elevation data, Water Resour. Res., 27(6), 1177-1191.

Moretti, G., and S. Orlandini (2008), Automatic delineation of drainage basins from contour elevation data using skeleton construction techniques, Water Resour. Res., 44, W05403, doi:10.1029/2007WR006309.

O'Callaghan, J., and D. M. Mark (1984), The extraction of drainage networks from digital elevation data, Comput. Vision Graphics Image Process., 28(3), 323-344.

Orlandini, S., and G. Moretti (2009a), Comment on "Global search algorithm for nondispersive flow path extraction" by Kyungrock Paik, $J$. Geophys. Res., 114, F04004, doi:10.1029/2008JF001193.

Orlandini, S., and G. Moretti (2009b), Determination of surface flow paths from gridded elevation data, Water Resour. Res., 45, W03417, doi: 10.1029/2008WR007099.

Orlandini, S., G. Moretti, M. Franchini, B. Aldighieri, and B. Testa (2003), Path-based methods for the determination of nondispersive drainage directions in grid-based digital elevation models, Water Resour. Res., 39(6), 1144, doi:10.1029/2002WR001639.

Orlandini, S., G. Moretti, M. A. Corticelli, P. E. Santangelo, A. Capra, R. Rivola, and J. D. Albertson (2012), Evaluation of flow direction methods against field observations of overland flow dispersion, Water Resour. Res., 48, W10523, doi:10.1029/2012WR012067.

Paik, K. (2008), Global search algorithm for nondispersive flow path extraction, J. Geophys. Res., 113, F04001, doi:10.1029/ 2007JF000964.

Quinn, P., K. Beven, P. Chevallier, and O. Planchon (1991), The prediction of hillslope flow paths for distributed hydrological modeling using digital terrain models, Hydrol. Processes, 5(1), 59-80

Seibert, J., and B. L. McGlynn (2007), A new triangular multiple flow direction algorithm for computing upslope areas from gridded digital elevation models, Water Resour. Res., 43, W04501, doi:10.1029/ 2006WR005128.

Tarboton, D. G. (1997), A new method for the determination of flow directions and upslope areas in grid digital elevation models, Water Resour. Res., 33(2), 309-319.

Taylor, A. E., and D. C. Lay (1980), Introduction to Functional Analysis, 467 pp., John Wiley, New York.

Vulikh, B. Z. (1963), Introduction to Functional Analysis for Scientists and Technologists, 404 pp., Pergamon, Oxford

Zhou, Q., P. Pilesjö, and Y. Chen (2011), Estimating surface flow paths on a digital elevation model using a triangular facet network, Water Resour. Res., 47, W07522, doi:10.1029/2010WR009961. 